\title{
DARK MATTER SCALING RELATIONS AND THE ASSEMBLY EPOCH OF COMA EARLY-TYPE GALAXIES
}

\author{
J. Thomas ${ }^{1,2}$, R. P. Saglia ${ }^{1,2}$, R. Bender ${ }^{1,2}$, D. Thomas ${ }^{3}$, K. Gebhardt $^{4}$, J. Magorrian $^{5}$, E. M. Corsini $^{6}$, And G. Wegner $^{7}$ \\ ${ }^{1}$ Universitätssternwarte München, Scheinerstraße 1, D-81679 München, Germany; jthomas@mpe.mpg.de \\ ${ }^{2}$ Max-Planck-Institut für Extraterrestrische Physik, Giessenbachstraße, D-85748 Garching, Germany \\ ${ }^{3}$ Institute of Cosmology and Gravitation, Mercantile House, University of Portsmouth, Portsmouth, PO1 2EG, UK \\ ${ }^{4}$ Department of Astronomy, University of Texas at Austin, C1400, Austin, TX78712, USA \\ ${ }^{5}$ Theoretical Physics, Department of Physics, University of Oxford, 1 Keble Road, OX1 3NP, Oxford, UK \\ ${ }^{6}$ Dipartimento di Astronomia, Università di Padova, vicolo dell'Osservatorio 3, I-35122 Padova, Italy \\ ${ }^{7}$ Department of Physics and Astronomy, 6127 Wilder Laboratory, Dartmouth College, Hanover, NH 03755-3528, USA \\ Received 2008 March 25; accepted 2008 September 21; published 2009 January 19
}

\begin{abstract}
Axisymmetric, orbit-based dynamical models are used to derive dark matter scaling relations for Coma earlytype galaxies. From faint to bright galaxies, halo core radii and asymptotic circular velocities increase. Compared to spirals of the same brightness, the majority of Coma early-type galaxies-those with old stellar populations - have similar halo core radii but more than two times larger asymptotic halo velocities. The average dark matter density inside $2 r_{\text {eff }}$ decreases with increasing luminosity and is 6.8 times larger than in disk galaxies of the same $B$-band luminosity. Compared at the same stellar mass, dark matter densities in ellipticals are 13.5 times higher than in spirals. Different baryon concentrations in ellipticals and spirals cannot explain the higher dark matter density in ellipticals. Instead, the assembly redshift $(1+z)$ of Coma early-type halos is likely about two times larger than of comparably bright spirals. Assuming that local spirals typically assemble at a redshift of one, the majority of bright Coma early-type galaxy halos must have formed around $z \approx 2-3$. For about half of our Coma galaxies, the assembly redshifts match with constraints derived from stellar populations. We find dark matter densities and estimated assembly redshifts of our observed Coma galaxies in reasonable agreement with recent semi-analytic galaxy formation models.
\end{abstract}

Key words: dark matter - galaxies: elliptical and lenticular, cD - galaxies: formation - galaxies: halos - galaxies: kinematics and dynamics

Online-only material: color figures

\section{INTRODUCTION}

Present day elliptical galaxies are known to host mostly old stellar populations (Trager et al. 2000; Terlevich \& Forbes 2002; Thomas et al. 2005a). Whether their stars have formed in situ or whether ellipticals assembled their present day morphology only over time (for example by mergers) is less clear. An important clue on the assembly redshift of a galaxy is provided by its dark matter density. For example, in the simple spherical collapse model (Gunn \& Gott 1972), the average density of virialized halos is proportional to the mean density of the universe at the formation epoch: halos which form earlier become denser. Similarly, in cosmological $N$-body simulations, the concentration (and, thus, the inner density) is found to be higher in halos that have assembled earlier (e.g., Navarro et al. 1996; Wechsler et al. 2002). In addition to this connection between formation epoch and halo density, the final halo mass distribution also depends on the interplay between dark matter and baryons during the actual galaxy formation process (e.g., Blumenthal et al. 1986; Binney et al. 2001). Then, the properties of galaxy halos provide valuable information about when and how a galaxy has assembled its baryons.

Despite its cosmological relevance, the radial distribution of dark (and luminous) mass in early-type galaxies is not well known: because of the lack of cold gas as a dynamical tracer, masses are difficult to determine. Stellar dynamical models require the exploration of a galaxy's orbital structure and have only recently become available for axisymmetric or more general systems (Cretton et al. 1999; Gebhardt et al. 2000; Thomas et al. 2004; Valluri et al. 2004; Cappellari et al. 2006;
De Lorenzi et al. 2007; Chanamé et al. 2008; van den Bosch et al. 2008). Scaling relations for the inner dark matter distribution have by now only been reported for round and nonrotating galaxies (Kronawitter et al. 2000; Gerhard et al. 2001) and spirals (Persic et al. 1996a, 1996b; Kormendy \& Freeman 2004). The aim of the present paper is to provide empirical scaling relations for generic cluster early-type galaxies (flattened, with different degrees of rotation). In particular, this paper is focussed on the inner dark matter density and its implications on the assembly redshift of elliptical galaxy halos.

The paper is organized as follows. In Section 2, we review the galaxy sample and its modeling. Dark matter scaling relations are presented in Section 3. Section 4 is dedicated to the dark matter density. The effect of baryons on the dark matter density is discussed in Section 5, while Section 6 deals with the halo assembly redshift. In Section 7, our results are compared to semi-analytic galaxy formation models. A summary is given in Section 8. In the following, we assume that the Coma cluster is at a distance of $d=100 \mathrm{Mpc}$.

\section{GALAXY SAMPLE, MODELS, AND BASIC DEFINITIONS}

The dark halo parameters discussed in this paper are derived from the axisymmetric, orbit-based dynamical models of bright Coma galaxies presented in Thomas et al. (2007a). The original sample comprises two cD galaxies, nine ordinary giant ellipticals and six lenticular/intermediate type galaxies with luminosities between $M_{B}=-18.79$ and $M_{B}=-22.56$. The spectroscopic and photometric observations are discussed 
in Jørgensen et al. (1996), Mehlert et al. (2000), Wegner et al. (2002), and Corsini et al. (2008). Our implementation of Schwarzschild's (1979) orbit superposition technique for axisymmetric potentials is described in Thomas et al. (2004, $2005 \mathrm{~b})$. For a detailed discussion of all the galaxy models, the reader is referred to Thomas et al. (2007a).

Three of the 17 galaxies from Thomas et al. (2007a) are excluded from the analysis below. Firstly, we do not consider the two central cD galaxies (GMP 2921 and GMP 3329; GMP numbers from Godwin et al. 1983) because their dark matter profiles may be affected by the cluster halo. Secondly, we omit the E/S0 galaxy GMP 1990 whose mass-to-light ratio is constant out to $3 r_{\text {eff }}$. The galaxy either has no dark matter within this radius, or its dark matter density follows the stellar light profile more closely than in any other Coma galaxy. In either case, the mass structure of this object is distinct from the rest of the sample galaxies. In addition to the remaining 14 galaxies, we consider two other Coma galaxies for which we collected data recently (GMP 3414, GMP 4822). The models of these galaxies are summarized in the Appendix.

Similar dynamical models as used here have been applied to the inner regions of ellipticals, where it has been assumed that mass follows light (e.g., Gebhardt et al. 2003; Cappellari et al. 2006). In contrast, our models explicitly include a dark matter component (see Thomas et al. 2007a). We probed for two parametric profiles. The first profiles were logarithmic halos

$$
\rho_{\mathrm{DM}}(r)=\frac{v_{h}^{2}}{4 \pi G} \frac{3 r_{h}^{2}+r^{2}}{\left(r_{h}^{2}+r^{2}\right)^{2}}
$$

which possess a constant-density core of size $r_{h}$ and have an asymptotically constant circular velocity $v_{h}$. The central density of these halos reads

$$
\rho_{h}=\frac{3 v_{h}^{2}}{4 \pi G r_{h}^{2}} .
$$

The second profiles were Navarro-Frenk-White (NFW) profiles

$$
\rho_{\mathrm{DM}}(r) \propto \frac{1}{r\left(r+r_{s}\right)^{2}},
$$

which are found in cosmological $\mathrm{N}$-body simulations (Navarro et al. 1996). The majority of Coma galaxies are better fit with logarithmic halos, but the significance over NFW halo profiles is marginal. Even if the best fit is obtained with an NFW halo, then the inner regions are still dominated by stellar mass (see Thomas et al. 2007a). In this sense, our models maximize the (inner) stellar mass.

In Section 3, we will only discuss results based on logarithmic halo fits (i.e., we use the halo parameters from Columns 5 and 6 in Table 2 of Thomas et al. 2007a and Columns 3 and 4 in Table 1). While these are not necessarily the more realistic profiles, they minimize systematics in the comparison with published scaling relations for spirals that were performed using cored profiles similar to our logarithmic halos. The NFW fits are used in Section 5.

The $B$-band luminosities of Coma galaxies used in this paper are taken from Hyperleda. We adopt a standard uncertainty of $\Delta M_{B}=0.3$ to account for zero-point uncertainties, systematic errors in the sky subtraction, seeing convolution, profile extrapolation, and others. Effective radii are taken from Jørgensen et al. (1995) and Mehlert et al. (2000). Here, we estimate the

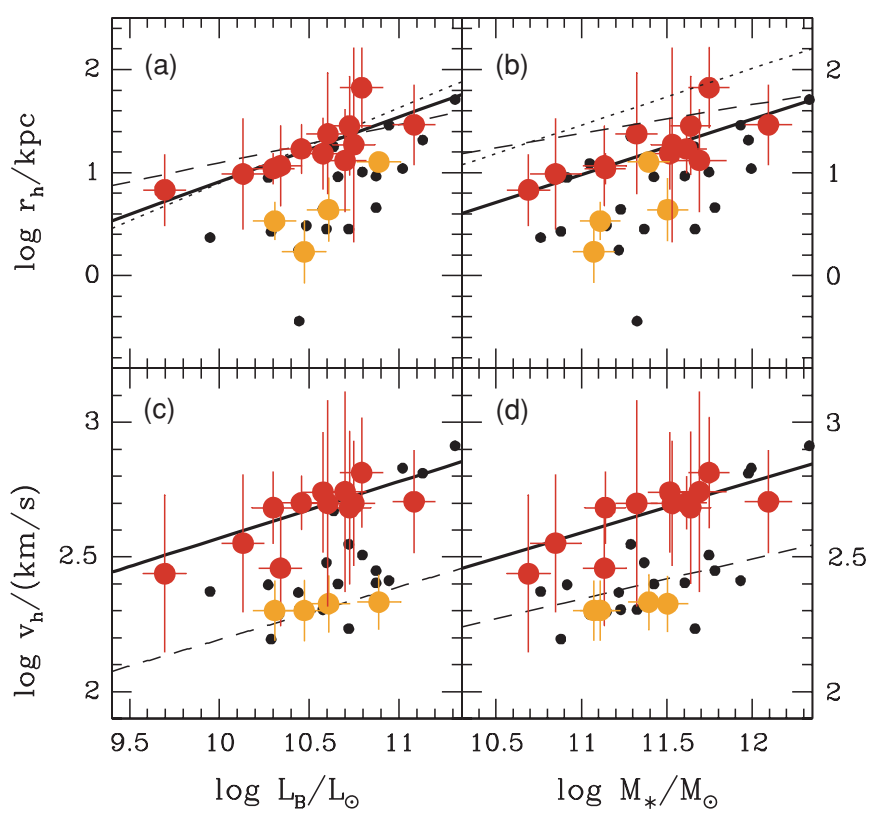

Figure 1. Halo core radius $r_{h}$ and circular velocity $v_{h}$ vs. $B$-band luminosity $L_{B}(a, c)$ and vs. stellar mass $M_{*}$ (b,d). Large symbols: Coma ellipticals (red: central stellar population age $\tau_{0}>6 \mathrm{Gyr}$, orange: $\tau_{0}<6 \mathrm{Gyr}$, details in the text); thick solid lines: fits to galaxies with $\tau_{0}>6 \mathrm{Gyr}$; small symbols: round early-type galaxies from Gerhard et al. (2001); dotted: spiral galaxy scaling relations from Persic et al. (1996a, 1996b); short-dashed: spiral galaxy scaling relations from Kormendy \& Freeman (2004).

(A color version of this figure is available in the online journal.)

Table 1

Model Parameters for GMP 3414 and GMP 4822

\begin{tabular}{lccccccc}
\hline \hline Galaxy & Fit & $\Upsilon$ & $r_{h}$ & $v_{h}$ & $c$ & $q$ & $\chi^{2}$ \\
$(1)$ & $(2)$ & $(3)$ & $(4)$ & $(5)$ & $(6)$ & $(7)$ & $(8)$ \\
\hline GMP 3414 & SC & 6.0 & & & & & 0.490 \\
& LOG & 4.5 & 9.7 & 356 & & & 0.239 \\
& NFW & 4.0 & & & 15.30 & 1.0 & 0.238 \\
\hline GMP 4822 & SC & 6.5 & & & & & 0.259 \\
& LOG & 5.5 & 13.1 & 552 & & & 0.229 \\
& NFW & 5.0 & & & 6.71 & 1.0 & 0.232 \\
\hline
\end{tabular}

Notes. (1) Galaxy ID; (2) type of fit (SC: without dark matter; LOG: logarithmic halo; NFW: halo profile from Navarro et al. 1996); (3) best-fit stellar $\Upsilon\left(M_{\odot} / L_{\odot}\right)$ ( $R_{C}$-band); (4) and (5) best-fit logarithmic halo parameters $r_{h}(\mathrm{kpc})$ and $v_{h}$ $\left(\mathrm{km} \mathrm{s}^{-1}\right)$; (6) and (7) best-fit NFW concentration $c$ and flattening $q$ (see Thomas et al. 2007a for details); (8) achieved goodness-of-fit $\chi^{2}$ (per data point).

errors to be $\Delta \log r_{\text {eff }}=0.1$. This is slightly higher than the uncertainties given in Jørgensen et al. (1995), but accounts for possible systematic errors (Saglia et al. 1997). Stellar masses were computed from our best-fit stellar mass-to-light ratios $\Upsilon$ and $R$-band luminosities of Mehlert et al. (2000). In case the best fit is obtained with a logarithmic halo, $\Upsilon$ is taken from column 4 of Table 2 in Thomas et al. (2007a). In case of an NFW fit, $\Upsilon$ comes from column 8 of the same table. The best-fit stellar mass-to-light ratios of GMP 3414 and GMP 4822 are given in column 1 of Table 1.

\section{DARK MATTER SCALING RELATIONS}

Figure 1 shows the scalings of dark halo core radii $r_{h}$ and halo asymptotic circular velocities $v_{h}$ with $B$-band luminosity $L_{B}$ and stellar mass $M_{*}$ (the corresponding galaxy parameters with errors are listed in Table 2). Both halo core sizes and halo circular velocities tend to increase with luminosity and mass. The case 
Table 2

Galaxy Parameters Shown in Figure 1

\begin{tabular}{lcccc}
\hline \hline $\begin{array}{l}\text { Galaxy } \\
(1)\end{array}$ & $\begin{array}{c}\log \frac{L_{B}}{L_{\odot}} \\
(2)\end{array}$ & $\begin{array}{c}\log \frac{M_{*}}{M_{\odot}} \\
(3)\end{array}$ & $\begin{array}{c}\log \frac{r_{h}}{\mathrm{kpc}} \\
(4)\end{array}$ & $\begin{array}{c}\log \frac{v_{h}}{\mathrm{~km} \mathrm{~s}^{-1}} \\
(5)\end{array}$ \\
\hline 0144 & 10.61 & $11.56 \pm 0.12$ & $0.64 \pm 0.31$ & $2.33 \pm 0.10$ \\
0282 & 10.46 & $11.60 \pm 0.12$ & $1.23 \pm 0.24$ & $2.70 \pm 0.10$ \\
0756 & 10.89 & $11.13 \pm 0.12$ & $1.10 \pm 0.09$ & $2.33 \pm 0.10$ \\
1176 & 10.31 & $10.73 \pm 0.13$ & $0.53 \pm 0.18$ & $2.30 \pm 0.11$ \\
1750 & 10.75 & $11.58 \pm 0.12$ & $1.27 \pm 0.95$ & $2.70 \pm 0.23$ \\
2417 & 10.60 & $11.43 \pm 0.12$ & $1.38 \pm 0.59$ & $2.70 \pm 0.38$ \\
2440 & 10.30 & $11.23 \pm 0.12$ & $1.04 \pm 0.15$ & $2.68 \pm 0.13$ \\
3414 & 10.13 & $11.02 \pm 0.15$ & $0.99 \pm 0.54$ & $2.55 \pm 0.26$ \\
3510 & 10.34 & $11.28 \pm 0.13$ & $1.07 \pm 0.39$ & $2.46 \pm 0.21$ \\
3792 & 10.58 & $11.56 \pm 0.13$ & $1.18 \pm 0.35$ & $2.74 \pm 0.22$ \\
3958 & 9.70 & $10.81 \pm 0.13$ & $0.83 \pm 0.35$ & $2.44 \pm 0.29$ \\
4822 & 10.70 & $11.69 \pm 0.16$ & $1.11 \pm 0.50$ & $2.74 \pm 0.37$ \\
4928 & 11.08 & $12.06 \pm 0.14$ & $1.46 \pm 0.39$ & $2.71 \pm 0.19$ \\
5279 & 10.72 & $11.59 \pm 0.12$ & $1.45 \pm 0.48$ & $2.68 \pm 0.28$ \\
5568 & 10.79 & $11.89 \pm 0.12$ & $1.82 \pm 0.39$ & $2.81 \pm 0.20$ \\
5975 & 10.47 & $11.04 \pm 0.12$ & $0.23 \pm 0.30$ & $2.30 \pm 0.11$
\end{tabular}

Notes. (1) Galaxy ID from Godwin et al. (1983); (2) galaxy $B$-band luminosity $L_{B}$; (3) stellar mass $M_{*}$ in solar units; (4) and (5) logarithmic halo core radius $\log r_{h} / \mathrm{kpc}$ and circular velocity $\log v_{h} / \mathrm{km} \mathrm{s}^{-1}$.

for a correlation between $v_{h}$ and $L_{B}$ is weak, if the sample as a whole is considered (see column 8 of Table 3 ). However, four galaxies (GMP 0144, GMP 0756, GMP 1176 and GMP 5975) separate from the rest of the sample galaxies in having both noticeably smaller $r_{h}$ and $v_{h}$. These galaxies are shown in light color in Figure 1. As a general trend, halo parameters tend to scale more tightly with luminosity and mass when these galaxies are omitted. The solid lines in Figure 1 show corresponding loglinear fits ${ }^{8}$. For comparison, in Table 3 we give both, fits to all Coma galaxies as well as fits to the subsample without the four galaxies offset in Figure 1. The difference between these four galaxies and the rest of the sample is further discussed below.

The logarithmic halos of Equation (1) have two free parameters. Any pair of $r_{h}, v_{h}$ or $\rho_{h}$ characterizes a specific halo. Figure 2 shows a plot of $\rho_{h}$ versus $r_{h}$. Both halo parameters are clearly correlated. A linear relation fits the points with a minimum $\chi_{\text {red }}^{2}=0.41$ (per degree of freedom; see Table 3). This rather low value partly derives from a degeneracy between the halo parameters in the dynamical modeling (e.g., Gerhard et al. 1998; Thomas et al. 2004) which correlates the errors in both quantities. In Table 3, such a correlation between the errors is not taken into account and the $\chi_{\text {red }}^{2}$ might thus be underestimated. A $\chi_{\text {red }}^{2}$ much larger than unity would indicate some intrinsic scatter in Figure 2, whereas the low $\chi_{\text {red }}^{2}$ quoted in Table 3 formally rules out any intrinsic scatter. Note that dark matter halos in cosmological $N$-body simulations can be approximated by a two-parameter family of halo models, where the parameters are correlated qualitatively in a similar way as revealed by Figure 2 (e.g., Navarro et al. 1996; Wechsler et al. 2002), but with some intrinsic scatter.

The four galaxies offset in Figure 1 are also slightly offset in Figure 2. However, given the large uncertainties, this is not significant and Figure 2 is consistent with the halos of the four galaxies belonging to the same one-parameter family as established by the remaining Coma galaxies. This implies that the four galaxies primarily differ in the amount of stellar light

\footnotetext{
8 Fits for this paper are performed with the routine fitexy of Press et al. (1992).
}

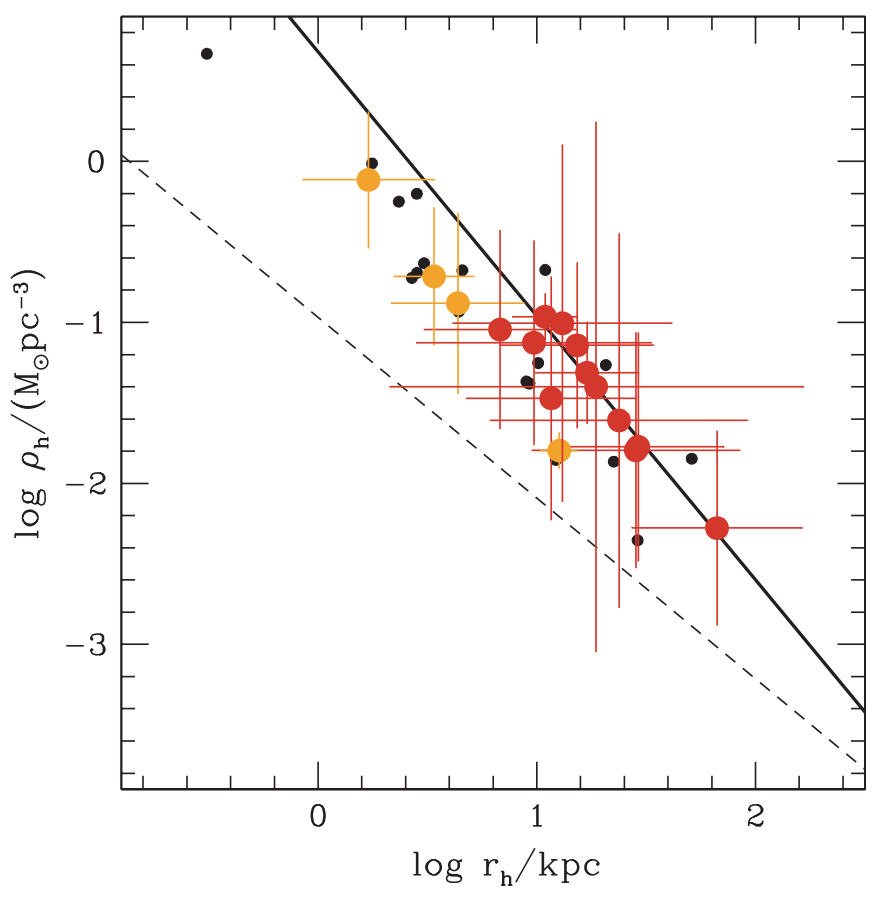

Figure 2. Central dark matter density $\rho_{h}$ vs. halo core radius $r_{h}$. Symbols and lines as in Figure 1.

(A color version of this figure is available in the online journal.)

(and stellar mass, respectively) that is associated with a given halo. It is noteworthy that the four galaxies have stellar ages $\tau_{0}<6$ Gyr (Mehlert et al. 2003), while all other Coma galaxies are significantly older (mostly $\tau_{0} \gtrsim 10 \mathrm{Gyr}$ ). A mere stellar population effect, however, is unlikely to explain the offset of the four galaxies. In this case, differences to other Coma galaxies should vanish when galaxies are compared at the same stellar mass, which is not consistent with Figures 1(b) and 1(d). It should be noted, though, that the stellar masses used here are taken from the dynamical models. A detailed comparison with mass-to-light ratios from stellar population synthesis models is planed for a future publication (J. Thomas et al. 2009, in preparation).

In Figure 3(a), we plot $r_{h}$ against $r_{\text {eff }}$. Larger core radii are found in more extended galaxies. Three of the four galaxies with young cores are again offset. Note that these three galaxies have similar $r_{\text {eff }}$ than other Coma galaxies of the same luminosity (see Figure 3(b)). This makes a higher baryon concentration unlikely to be the cause of their small halo core radii. An exceptional case is GMP 0756: it has a small core radius, a ratio $r_{h} / r_{\text {eff }}$ which is typical for Coma galaxies with old stellar populations and a relatively small $r_{\text {eff }}$. The scalings of $r_{\text {eff }}$ and $r_{h}$ with luminosity in Coma galaxies with old stellar populations imply a roughly constant ratio $r_{h} / r_{\text {eff }} \approx 3$. Moreover, disk galaxies of the same luminosity show a similar ratio.

The fact that all four galaxies offset in Figures 1 and 3 appear at projected cluster-centric distances $D>1 \mathrm{Mpc}$, and have young stellar populations suggests that they may have entered the Coma cluster only recently. We will discuss this point in Section 3.1.

\subsection{Comparison with Round and Nonrotating Early-Type Galaxies}

Kronawitter et al. (2000) and Gerhard et al. (2001) studied dark matter halos of 21 nearly round (E0-E2) and nonrotating 
Table 3

Dark Matter Scaling Relations

\begin{tabular}{|c|c|c|c|c|c|c|c|c|}
\hline \multicolumn{2}{|c|}{ Relation } & \multicolumn{2}{|c|}{$\log y=a+b \log x$} & \multirow{2}{*}{$\begin{array}{l}\chi_{\text {red }}^{2} \\
\text { (5) } \\
\end{array}$} & \multirow{2}{*}{$\begin{array}{l}\mathrm{rms} \\
(6) \\
\end{array}$} & \multirow{2}{*}{$\begin{array}{c}\langle\Delta \log y\rangle \\
(7)\end{array}$} & \multirow{2}{*}{$\begin{array}{l}\mathcal{P} \\
(8) \\
\end{array}$} & \multirow{2}{*}{$\begin{array}{l}\text { Figure } \\
\text { (9) }\end{array}$} \\
\hline $\begin{array}{l}y \\
\text { (1) } \\
\end{array}$ & $\begin{array}{c}x \\
(2) \\
\end{array}$ & $\begin{array}{c}a \\
(3) \\
\end{array}$ & $\begin{array}{c}b \\
(4) \\
\end{array}$ & & & & & \\
\hline \multicolumn{9}{|c|}{ Fits to all galaxies } \\
\hline$\frac{r_{h}}{\mathrm{kpc}}$ & $\frac{L_{B}}{10^{11} L_{\odot}}$ & $1.24 \pm 0.14$ & $0.55 \pm 0.26$ & 1.24 & 0.35 & 0.39 & 0.010 & \\
\hline$\frac{r_{h}}{\mathrm{kpc}}$ & $\frac{M_{*}}{10^{11} M_{\odot}}$ & $0.71 \pm 0.12$ & $0.90 \pm 0.28$ & 0.85 & 0.31 & 0.39 & 0.002 & \\
\hline$\frac{v_{h}}{\mathrm{~km} \mathrm{~s}^{-1}}$ & $\frac{L_{B}}{10^{11} L_{\odot}}$ & $0.52 \pm 0.12$ & $0.07 \pm 0.23$ & 1.62 & 0.19 & 0.21 & 0.109 & \\
\hline$\frac{v_{h}}{\mathrm{~km} \mathrm{~s}^{-1}}$ & $\frac{M_{*}}{10^{11} M_{\odot}}$ & $0.33 \pm 0.07$ & $0.45 \pm 0.16$ & 1.04 & 0.17 & 0.21 & 0.002 & \\
\hline$\frac{\rho_{h}}{M_{\odot}^{3}}$ & $\frac{r_{h}}{\mathrm{kpc}}$ & $0.67 \pm 0.64$ & $-1.99 \pm 0.57$ & 0.41 & 0.41 & 0.66 & 0.001 & \\
\hline$\frac{r_{h}}{\mathrm{kpc}}$ & $\frac{r_{\text {eff }}}{\mathrm{kpc}}$ & $0.48 \pm 0.23$ & $0.79 \pm 0.32$ & 1.15 & 0.31 & 0.39 & 0.026 & \\
\hline$\frac{\rho_{h}}{M_{\odot}^{3}}$ & $\frac{L_{B}}{10^{11} L_{\odot}}$ & $-1.87 \pm 0.18$ & $-1.28 \pm 0.33$ & 0.75 & 0.47 & 0.66 & 0.019 & 4(a) \\
\hline$\frac{\rho_{h}}{M_{\odot}^{3}}$ & $\frac{M_{*}}{10^{11} M_{\odot}}$ & $-0.77 \pm 0.19$ & $-1.57 \pm 0.38$ & 0.94 & 0.52 & 0.66 & 0.058 & 4(b) \\
\hline$\frac{\left\langle\rho_{\mathrm{DM}}\right\rangle}{M_{\odot}^{3}}$ & $\frac{L_{B}}{10^{11} L_{\odot}}$ & $-2.36 \pm 0.14$ & $-1.56 \pm 0.24$ & 1.31 & 0.38 & 0.32 & 0.004 & 4(c) \\
\hline$\frac{\left\langle\rho_{\mathrm{DM}}\right\rangle}{M_{\odot}^{3}}$ & $\frac{M_{*}}{10^{11} M_{\odot}}$ & $-1.10 \pm 0.11$ & $-1.57 \pm 0.24$ & 1.79 & 0.44 & 0.32 & 0.025 & $4(d)$ \\
\hline \multicolumn{9}{|c|}{ Fits omitting galaxies with young stellar cores } \\
\hline$\frac{r_{h}}{\mathrm{kpc}}$ & $\frac{L_{B}}{10^{11} L_{\odot}}$ & $1.54 \pm 0.21$ & $0.63 \pm 0.33$ & 0.16 & 0.16 & 0.44 & 0.002 & 1(a) \\
\hline$\frac{r_{h}}{\mathrm{kpc}}$ & $\frac{M_{*}}{10^{11} M_{\odot}}$ & $0.98 \pm 0.14$ & $0.54 \pm 0.29$ & 0.18 & 0.17 & 0.44 & 0.007 & 1 (b) \\
\hline$\frac{v_{h}}{\mathrm{~km} \mathrm{~s}^{-1}}$ & $\frac{L_{B}}{10^{11} L_{\odot}}$ & $0.78 \pm 0.11$ & $0.21 \pm 0.21$ & 0.14 & 0.07 & 0.24 & 0.016 & $1(\mathrm{c})$ \\
\hline$\frac{v_{h}}{\mathrm{~km} \mathrm{~s}^{-1}}$ & $\frac{M_{*}}{10^{11} M_{\odot}}$ & $0.59 \pm 0.10$ & $0.19 \pm 0.17$ & 0.12 & 0.07 & 0.24 & 0.008 & $1(\mathrm{~d})$ \\
\hline$\frac{\rho_{h}}{M_{\odot}^{3}}$ & $\frac{r_{h}}{\mathrm{kpc}}$ & $0.68 \pm 1.35$ & $-1.64 \pm 0.96$ & 0.04 & 0.18 & 0.75 & 0.006 & 2 \\
\hline$\frac{r_{h}}{\mathrm{kpc}}$ & $\frac{r_{\text {eff }}}{\mathrm{kpc}}$ & $0.79 \pm 0.21$ & $0.62 \pm 0.30$ & 0.10 & 0.14 & 0.44 & 0.017 & 3 \\
\hline$\frac{\rho_{h}}{M_{\odot}^{3}}$ & $\frac{L_{B}}{10^{11} L_{\odot}}$ & $-1.81 \pm 0.36$ & $-1.02 \pm 0.54$ & 0.31 & 0.34 & 0.75 & 0.028 & \\
\hline$\frac{\rho_{h}}{M_{\odot}^{3}}$ & $\frac{M_{*}}{10^{11} M_{\odot}}$ & $-0.94 \pm 0.17$ & $-0.81 \pm 0.43$ & 0.31 & 0.36 & 0.75 & 0.090 & \\
\hline$\frac{\left\langle\rho_{\mathrm{DM}}\right\rangle}{M_{\odot}^{3}}$ & $\frac{L_{B}}{10^{11} L_{\odot}}$ & $-2.01 \pm 0.20$ & $-1.04 \pm 0.30$ & 0.77 & 0.31 & 0.41 & 0.012 & \\
\hline$\frac{\left\langle\rho_{\mathrm{DM}}\right\rangle}{M_{\odot}^{3}}$ & $\frac{M_{*}}{10^{11} M_{\odot}}$ & $-1.12 \pm 0.11$ & $-0.74 \pm 0.25$ & 0.86 & 0.33 & 0.41 & 0.020 & \\
\hline
\end{tabular}

Notes. (1) and (2) Fitted quantities; (3) and (4) parameters of linear fit with errors; (5) reduced $\chi_{\text {red }}^{2}$ of the fit; (6) rms scatter in $\log y$; (7) mean error $\langle\Delta \log y\rangle$; (8) significance of the relation (probability $\mathcal{P}$ that there is no relation according to a Spearman rank order correlation test); (9) figure in which the relation is shown.

galaxies (K2000 in the following). K2000 galaxies have similar luminosities $M_{B}$ and half-light radii $r_{\text {eff }}$ as ours, but the K2000 sample contains a mixture of field ellipticals and galaxies in the Virgo and Fornax clusters. The dynamical models of Kronawitter et al. (2000) differ in some respects from the ones described in Section 2 and this will be further discussed later. However, in their mass decomposition, Kronawitter et al. (2000) assumed the same halo profile as in Equation (1), such that we can directly compare their halo parameters to ours (see small black dots in Figures 1-3).

We find halo parameters of both samples in the same range, but Coma galaxies of the same $L_{B}$ have on average larger halo core radii than K2000 galaxies. In fact, K2000 galaxies populate a wider range of $r_{h}$ than (old) Coma galaxies. However, the halos of the K2000 galaxies themselves are not different from the ones around Coma early-type galaxies, as both belong to the same one-parameter family (see Figure 2). The main difference is that K2000 galaxies are brighter (and have higher stellar mass) than Coma early-type galaxies with a similar halo. Can this be an artifact related to differences in the dynamical models?

Many of the K2000 models are based on $B$-band photometry, while we used $R_{C}$-band images for the Coma galaxies. Elliptical galaxies become bluer toward the outer parts and $B$-band light profiles are slightly shallower than $R$-band profiles. Likewise, mass profiles of galaxies are generally shallower than their light profiles, such that there might be less need for dark matter in $B$-band models than in $R$-band models. Kronawitter et al. (2000) checked for this by modeling one galaxy (NGC 3379) in both bands and found comparable results. The photometric data are therefore unlikely to cause differences between the two samples.

K2000 galaxies were modeled assuming spherical symmetry. Not all apparently round galaxies need to be intrinsically spherical. Neglecting the flattening along the line-of-sight can result in an underestimation of a galaxy's mass (e.g., Thomas et al. 2007b). Based on the average intrinsic flattening of ellipticals in the luminosity interval of interest here, Kronawitter et al. (2000) estimated that the assumption of spherical symmetry should affect mass-to-light ratios only at the $10 \%$ level. We expect the effect on $v_{h}$ to be correspondingly small. In addition, it is not obvious why spherical symmetry should enforce systematically small $r_{h}$, such that the different symmetry assumptions are also unlikely to explain the more extended cores and higher circular velocities in Coma galaxy halos.

Since the shape of a galaxy is related to its evolutionary history, the round and nonrotating K2000 galaxies could be intrinsically different from the mostly flattened and rotating Coma galaxies. Structural differences could also be related to the fact that K2000 galaxies are located in a variety of environments, with less galaxies in high-density regions like Coma. For example, stellar population models indicate that field 


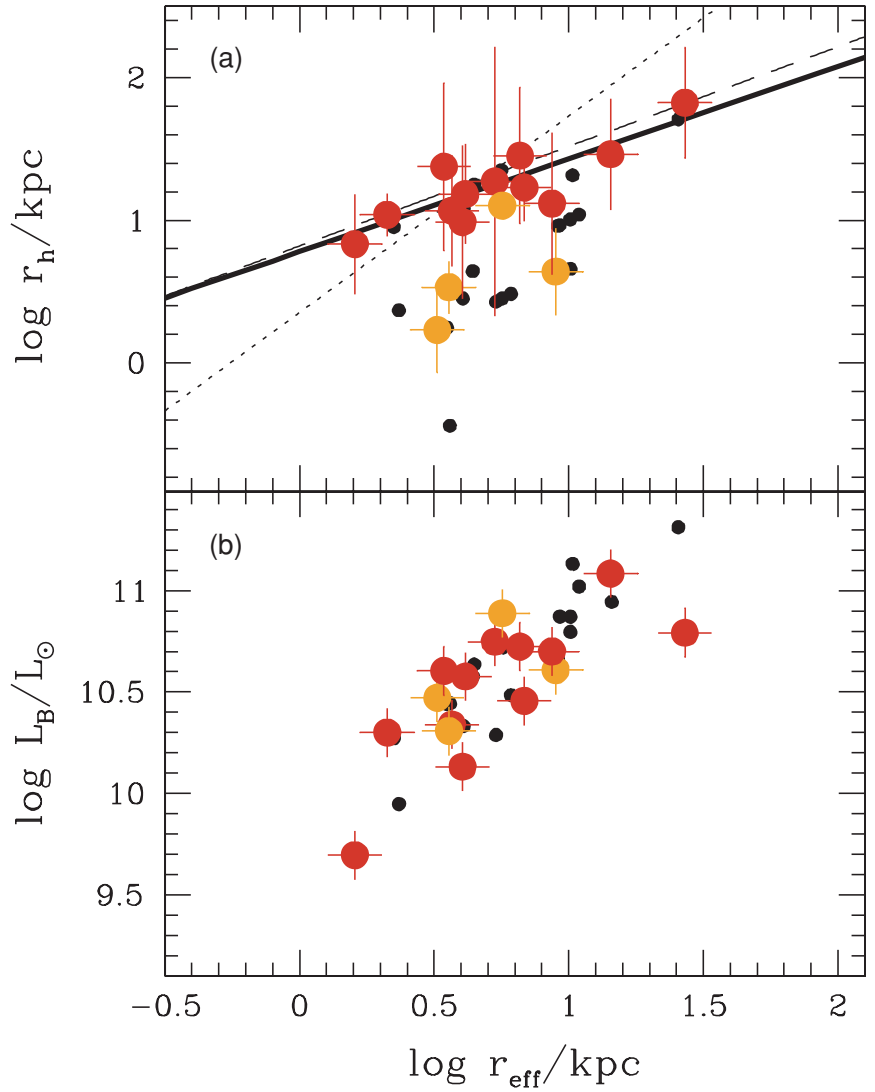

Figure 3. Halo core radius $r_{h}(a)$ and $B$-band luminosity $(b)$ vs. effective radius $r_{\text {eff }}$. Large symbols: Coma galaxies; small dots: round galaxies from Gerhard et al. (2001). Lines in panel (a) as in Figure 1.

(A color version of this figure is available in the online journal.)

ellipticals are on average younger and have more extended star formation histories than cluster galaxies (Thomas et al. 2005a). But a mere difference in the stellar populations cannot explain the difference between K2000 and Coma galaxies, as in this case the scaling relations with respect to $M_{*}$ should be similar in both samples. This is ruled out by Figure 1(b) and (d).

It is noteworthy that most of the K2000 galaxies in Figures 1-3 appear similar to the four Coma galaxies with distinctly small $r_{h}$ and $v_{h}$. As has been discussed earlier, these galaxies may have entered the Coma cluster only recently and - in this respect - are more representative for a field galaxy population rather than being genuine old cluster galaxies. A consistent explanation for both, the offset between young and old Coma galaxies on the one hand and the difference between old Coma galaxies and K2000 galaxies on the other would then be that field galaxies have lower $r_{h}$ and $v_{h}$ than cluster galaxies of the same stellar mass. Because there are more field galaxies in the K2000 sample than in Coma, Coma galaxy halos would be expected to have on average larger cores and to be more massive (consistent with Figure 1). A larger comparison sample of field elliptical halos is required to conclude this point.

\subsection{Comparison with Spiral Galaxies}

Two independent derivations of dark matter scaling relations for spiral galaxies are included in Figures $1-3$ by the dotted and dashed lines. The dotted lines show scaling relations from Persic et al. (1996a, 1996b). They are based on maximum disk rotation curve decompositions with the halo density from Equation (1). Persic et al. (1996a, 1996b) give halo core radii scaled by the optical disk radius. To reconstruct the underlying relationship between $r_{h}$ and $L_{B}$, we follow Gerhard et al. (2001) and assume exponential disks with

$$
\left(\frac{r_{\mathrm{eff}}^{\mathrm{S}}}{\mathrm{kpc}}\right)=8.4\left(\frac{L_{B}}{10^{11} L_{\odot}}\right)^{0.53}
$$

(the empirical fit in Gerhard et al. 2001 has been transformed to our distance scale).

The dashed lines in Figures 1-3 fit the combined sample of 55 rotation curve decompositions of Kormendy \& Freeman (2004). These authors discuss various halo profiles, but we here consider only nonsingular isothermal dark matter halos. Though these are most similar to Equation (1), isothermal cores and circular velocities are not exactly identical as in logarithmic halos. To account for the difference, we fitted a logarithmic halo to a nonsingular isothermal density profile (see Table 4.1 of Binney $\&$ Tremaine 1987). The fit was restricted to the region with kinematic data (typically inside two core radii). We found that the logarithmic halo fit yields a 3\% larger core radius $r_{h}$ and a $10 \%$ smaller circular velocity $v_{h}$. The central halo density is reproduced to $0.001 \mathrm{dex}$ (not surprising given that the cores of the two profiles were matched). Thus, under the assumption that fits performed with the two profiles indeed match inside two core radii, a correction of the derived halo parameters is not needed. Scaling relations from Kormendy \& Freeman (2004) are shown in Figures 1 and 2 without any correction.

Persic et al. (1996a, 1996b) and Kormendy \& Freeman (2004) discuss the scaling of disk galaxy halos with $B$-band luminosity. In order to compare early-type galaxies and spirals also at the same stellar masses, we used

$$
\left(\frac{M_{*}}{L_{B}}\right)^{\mathrm{S}}=2 \times\left(\frac{L_{B}}{10^{11} L_{\odot}}\right)^{0.33}
$$

for the stellar mass-to-light ratios of disks. Equation (5) is derived from the Tully-Fisher and stellar-mass Tully-Fisher relations of Bell \& De Jong (2001).

Both in luminosity and in stellar mass, spirals and ellipticals follow similar global trends. However, while old Coma earlytype galaxies have halo core radii of similar size as spirals of the same $B$-band luminosity, the asymptotic halo circular velocities are 2.4 times higher than in corresponding spirals. In contrast, early-type galaxies with young central stellar populations have about 4 times smaller core radii than spirals, but similar asymptotic halo velocities. When galaxies are compared at the same stellar mass, then differences between ellipticals and spirals become larger $\left(60 \%\right.$ smaller $r_{h}$ and 1.8 times higher $v_{h}$ in old Coma early-type galaxies compared to spirals; $90 \%$ smaller $r_{h}$ and $20 \%$ smaller $v_{h}$ in Coma galaxies with young central stellar populations). In addition, the halos of early-type galaxies and spirals do not belong to the same one-parameter family (see Figure 2). At a given $r_{h}$, dark matter densities in ellipticals are about 0.5 dex higher than in spirals.

\section{THE DARK MATTER DENSITY}

Figure 4 shows scaling laws for dark matter densities. The central dark matter density $\rho_{h}$ (see Equation (2)) of the logarithmic halo fits is plotted in panels (a) and (b) versus luminosity and stellar mass. For panels (c) and (d), the bestfit dark matter halo of each galaxy (being either logarithmic or NFW) is averaged within $2 r_{\text {eff }}$

$$
\left\langle\rho_{\mathrm{DM}}\right\rangle \equiv \frac{3}{4 \pi} \frac{M_{\mathrm{DM}}\left(2 r_{\mathrm{eff}}\right)}{\left(2 r_{\mathrm{eff}}\right)^{3}}
$$




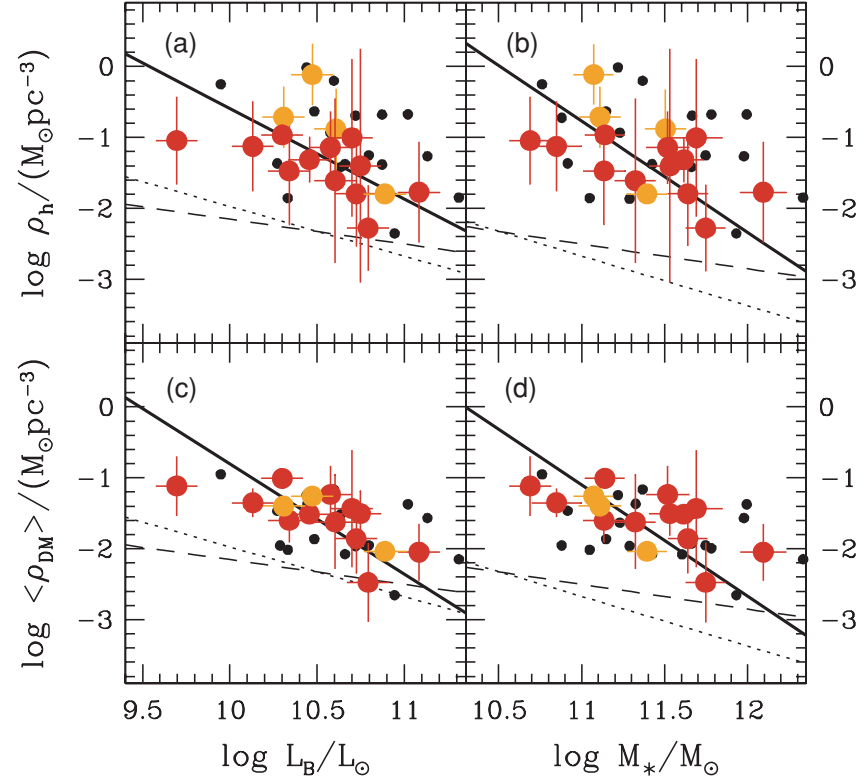

Figure 4. Central halo density $\rho_{h}$ and average dark matter density $\left\langle\rho_{\mathrm{DM}}\right\rangle$ inside $2 r_{\text {eff }}$ vs. luminosity $L_{B}(a, c)$ and stellar mass $M_{*}(b, d)$. Solid lines: fits including all Coma galaxies; dotted and dashed lines: spiral galaxy scaling relations as in Figure 1.

(A color version of this figure is available in the online journal.)

Here, $M_{\mathrm{DM}}(r)$ equals the cumulative dark mass inside a sphere with radius $r$. Average dark matter densities are quoted in column (2) of Table 4.

The general trend for both densities is to decrease with increasing galaxy luminosity. Thereby the central densities $\rho_{h}$ scatter more than the averaged $\left\langle\rho_{\mathrm{DM}}\right\rangle$. For two reasons, $\left\langle\rho_{\mathrm{DM}}\right\rangle$ quantifies the actual dark matter density more robustly than $\rho_{h}$. Firstly, our estimate of the very central dark matter density depends strongly on the assumed halo profile. Instead, averaged over $2 r_{\text {eff }}$ differences between logarithmic halo fits and fits with NFW profiles are small compared to the statistical errors (averaged over the Coma sample NFW fits yield 0.1-0.2 dex higher $\left\langle\rho_{\text {DM }}\right\rangle$ than fits with logarithmic halos).

Secondly, the most significant differences between $\rho_{h}$ and $\left\langle\rho_{\text {DM }}\right\rangle$ occur in the four Coma galaxies with distinct halos discussed in Section 3. These galaxies have young central stellar populations. If the bulk of stars in these galaxies is old, however, then the related radial increase of the stellar mass-to-light ratio could contribute to their small $r_{h}$ and large $\rho_{h}$. This is because in our models it is assumed that the stellar mass-to-light ratio is radially constant. By construction then, any increase in the mass-to-light ratio with radius (being either due to a stellar population gradient or due to dark matter) is attributed to the halo component. In galaxies with a significant increase of the stellar $M / L$ with radius, the "halo" component of the model thereby has to account for both, additional stellar and possible dark mass (Thomas 2006). Any contamination with stellar mass will be largest at small radii, where the increase in the stellar $M / L$ dominates the shape of the mass profile. The averaging radius in Equation (6) is therefore chosen as large as possible. The value of $2 r_{\text {eff }}$ is a compromise for the whole sample, because the kinematic data extend to $1-3 r_{\text {eff }}$ and the averaging should not go much beyond the last data point.

Compared to the Coma galaxies, the majority of K2000 galaxies have larger $\rho_{h}$. After averaging inside $2 r_{\text {eff }}$, the halo densities in both samples become comparable, however. In this respect, the K2000 galaxies again resemble the four Coma galaxies with young stellar cores.

The dotted and dashed lines in Figure 4 show spiral galaxies. Their halo densities need not to be averaged before comparison, because core sizes of spirals (in the considered luminosity interval) are larger than $2 r_{\text {eff }}$. Averaged over the whole sample, we find dark matter densities in Coma early-type galaxies a factor of 6.8 higher than in spirals of the same luminosity. Comparing early-type galaxies and spirals at the same stellar mass, then the overdensity amounts to a factor of 13.5. Does this imply that spirals and ellipticals of the same luminosity have formed in different dark matter halos?

\section{BARYONIC CONTRACTION}

Even if ellipticals and spirals would have formed in similar halos, the final dark matter densities after the actual galaxy formation process could be different, since the baryons in ellipticals and spirals are not distributed in the same way. This effect can be approximated as follows: assume that (a spherical) baryonic mass distribution $M_{*}(r)$ condenses slowly out of an original halo+baryon distribution $M_{i}(r)$. The halo responds adiabatically and contracts into the mass distribution $M_{\mathrm{DM}}(r)$. If the original particles move on circular orbits then

$$
r\left[M_{*}(r)+M_{\mathrm{DM}}(r)\right]=r_{i} M_{i}\left(r_{i}\right)
$$

turns out to be an adiabatic invariant (Blumenthal et al. 1986).

In case of the Coma galaxies, $M_{*}$ and $M_{\mathrm{DM}}$ are known from the dynamical modeling and Equation (7) can be solved for $M_{i}{ }^{9}$. It characterizes the original halo mass distribution before the actual galaxy formation. Had a disk with baryonic mass $M_{*}^{\mathrm{D}}$ grown in this original halo-instead of an early type galaxythen the halo contraction would have been different such that in general $M_{\mathrm{DM}}^{\mathrm{D}} \neq M_{\mathrm{DM}}$. The difference between $M_{\mathrm{DM}}^{\mathrm{D}}$ and $M_{\mathrm{DM}}$ actually determines how much dark matter densities of ellipticals and spirals would differ, if both had formed in the same original halos. To quantify this further, let us consider the spherically averaged mass distribution of a thin exponential disk for $M_{*}^{\mathrm{D}}$ (e.g., Blumenthal et al. 1986). It is fully determined by a scale radius and a mass. For a given Coma elliptical with luminosity $L_{B}$, the scale radius and the mass of a realistic disk with the same luminosity can be taken from Equations (4) and (5). Then, given $M_{*}^{\mathrm{D}}$ and $M_{i}$ (the recontracted Coma galaxy halo), Equation (7) can be solved for the baryon-contracted halo $M_{\mathrm{DM}}^{\mathrm{D}}$ around the comparison disk (see Blumenthal et al. 1986). Once $M_{\mathrm{DM}}^{\mathrm{D}}$ is known, the average $\left\langle\rho_{\mathrm{DM}}\right\rangle^{\mathrm{D}}$ follows directly.

If ellipticals and spirals (of the same $L_{B}$ ) would have formed in the same halos, then

$$
\delta_{\mathrm{bar}} \equiv \frac{\left\langle\rho_{\mathrm{DM}}\right\rangle}{\left\langle\rho_{\mathrm{DM}}\right\rangle^{\mathrm{D}}}
$$

should fully account for the observed ratio of elliptical to spiral dark matter densities. However, averaged over the Coma sample we find $\delta_{\text {bar }} \approx 2$ and, thus, that the higher baryon concentration in early-type galaxies is not sufficient to explain the factor of 6.8 between the dark matter densities of ellipticals and spirals at constant luminosity.

In general, the observed dark matter density ratio $\delta_{\text {obs }}$ between ellipticals and spirals will be a combination of a difference in the

\footnotetext{
9 Because of the large core radii in some galaxies (see Figures 1(c) and (d)), it is not always possible to find $M_{i}$ for logarithmic halos. Therefore, here we only consider the best-fit NFW halo of each galaxy.
} 
Table 4

Halo Densities, Halo Assembly Redshfits, and Stellar Ages

\begin{tabular}{lcccccc}
\hline \hline Galaxy & $\log \frac{\left\langle\rho_{\text {DM }}\right\rangle}{M_{\odot}^{-3}}$ & $z_{\text {form }}$ & $\log \frac{\left\langle\rho_{\text {DM }}\right\rangle / \delta_{\text {bar }}}{M_{\odot}^{-3}}$ & $z_{\text {form }}$ & $\begin{array}{c}\tau_{0} \\
\text { Gyr }\end{array}$ \\
$(1)$ & $(2)$ & $(3)$ & $(4)$ & $(5)$ & $(6)$ & $(7)$ \\
\hline 0144 & $-2.24 \pm 0.08$ & $1.23_{-0.56}^{+1.11}$ & $-2.00 \pm 0.08$ & $1.56_{-0.64}^{+1.56}$ & $5.8 \pm 0.5$ & $0.60_{-0.07}^{+0.08}$ \\
0282 & $-1.51 \pm 0.09$ & $2.66_{-0.92}^{+1.83}$ & $-1.98 \pm 0.09$ & $1.60_{-0.65}^{+1.30}$ & $7.7 \pm 0.8$ & $0.95_{-0.17}^{+0.20}$ \\
0756 & $-2.04 \pm 0.04$ & $1.92_{-0.73}^{+1.46}$ & $-1.89 \pm 0.04$ & $1.79_{-0.70}^{+1.40}$ & $3.1 \pm 0.2$ & $0.26_{-0.02}^{+0.02}$ \\
1176 & $-1.40 \pm 0.07$ & $2.77_{-0.94}^{+1.89}$ & $-1.61 \pm 0.07$ & $2.47_{-0.87}^{+1.74}$ & $3.3 \pm 0.4$ & $0.28_{-0.04}^{+0.04}$ \\
1750 & $-1.50 \pm 0.31$ & $3.15_{-1.04}^{+2.08}$ & $-1.80 \pm 0.31$ & $1.99_{-0.75}^{+1.50}$ & $11.3 \pm 1.7$ & $2.48_{-0.90}^{+2.90}$ \\
2417 & $-1.62 \pm 0.66$ & $2.57_{-0.89}^{+1.79}$ & $-2.01 \pm 0.66$ & $1.54_{-0.64}^{+1.27}$ & $11.5 \pm 2.4$ & $2.65_{-1.33}^{+\infty}$ \\
2440 & $-1.01 \pm 0.11$ & $4.07_{-1.27}^{+2.54}$ & $-1.69 \pm 0.11$ & $2.25_{-0.81}^{+1.63}$ & $13.5 \pm 2.1$ & $8.17_{-5.6}^{+\infty}$ \\
3414 & $-1.35 \pm 0.20$ & $2.64_{-0.91}^{+1.82}$ & $-1.60 \pm 0.20$ & $2.50_{-0.88}^{+1.75}$ & $11.2 \pm 2.7$ & $2.40_{-1.25}^{+\infty}$ \\
3510 & $-1.60 \pm 0.30$ & $2.27_{-0.82}^{+1.64}$ & $-2.02 \pm 0.30$ & $1.52_{-0.63}^{+1.26}$ & $14.2 \pm 1.6$ & $>4.26$ \\
3792 & $-1.24 \pm 0.40$ & $3.76_{-1.19}^{+2.38}$ & $-1.83 \pm 0.40$ & $1.93_{-0.73}^{+1.47}$ & $13.4 \pm 2.1$ & $7.33_{-4.84}^{+\infty}$ \\
3958 & $-1.11 \pm 0.42$ & $2.69_{-0.92}^{+1.84}$ & $-1.82 \pm 0.42$ & $1.97_{-0.74}^{+1.48}$ & $\ldots$ & $\cdots$ \\
4822 & $-1.43 \pm 0.83$ & $3.30_{-1.08}^{+2.15}$ & $-1.65 \pm 0.83$ & $2.40_{-0.77}^{+1.61}$ & $11.2 \pm 1.3$ & $2.40_{-0.77}^{+1.61}$ \\
4928 & $-2.05 \pm 0.40$ & $2.14_{-0.78}^{+1.57}$ & $-2.08 \pm 0.40$ & $1.42_{-0.61}^{+1.21}$ & $14.5 \pm 1.4$ & $>5.70$ \\
5279 & $-1.86 \pm 0.49$ & $2.13_{-0.78}^{+1.57}$ & $-2.08 \pm 0.49$ & $1.42_{-0.60}^{+1.21}$ & $10.9 \pm 0.8$ & $2.16_{-0.45}^{+0.67}$ \\
5568 & $-2.47 \pm 0.56$ & $1.01_{-0.50}^{+1.00}$ & $-2.40 \pm 0.56$ & $0.89_{-0.47}^{+0.95}$ & $9.6 \pm 0.6$ & $1.50_{-0.21}^{+0.26}$ \\
5975 & $-1.26 \pm 0.09$ & $3.49_{-1.12}^{+2.24}$ & $-1.52 \pm 0.09$ & $2.70_{-0.93}^{+1.85}$ & $5.7 \pm 1.2$ & $0.58_{-0.16}^{+0.20}$ \\
\hline
\end{tabular}

Notes. (1) Galaxy ID from Godwin et al. (1983); (2) average dark matter density $\left\langle\rho_{\mathrm{DM}}\right\rangle$ inside $2 r_{\text {eff }}$; (3) halo assembly redshift $z_{\text {form }}$ according to Equation (11) with $\delta=\delta_{\text {obs }}$ and $z_{\text {form }}^{S}=1$; (4) as column (2), but including the baryonic correction $\delta_{\text {bar }}$ defined in Equation (8) (we do not derive an error estimate for the baryonic contraction, but use the same errors in columns (2) and (4), respectively); (5) $z_{\text {form }}$ as in column (3), but with baryon corrected $\delta=\delta_{\text {halo }}$ and $z_{\text {form }}^{\mathrm{S}}(L)$ from Equation (12); (6) central stellar age $\tau_{0}$ from Table B.1 of Mehlert et al. (2003; GMP 3958 has no age estimate because of its very low $\mathrm{H} \beta$ ); (7) formation redshift $z\left(\tau_{0}\right)$ of the stars derived from column (6). In some galaxies, the stellar age or its upper limit exceeds the age of the universe in the adopted cosmology. In such cases, only a lower limit is given for $z\left(\tau_{0}\right)$ or the upper redshift error is set equal to $\infty$, respectively.

halo densities before baryon infall and a factor that comes from the baryons. Let $\delta_{\text {halo }}$ denote the baryon-corrected dark matter density ratio, then the simplest assumption is

$$
\delta_{\text {obs }}=\delta_{\text {bar }} \times \delta_{\text {halo }},
$$

with $\delta_{\text {bar }}$ from Equation (8). After applying this approximate baryon correction, dark matter densities in Coma ellipticals are still a factor of $\delta_{\text {halo }}=3.4$ higher than in spirals of the same luminosity. If the comparison is made at the same stellar mass, then $\delta_{\text {halo }}=6.4$. Note that our baryonic contraction corrections are likely upper limits, because in Equation (5) we only account for the stellar mass in the disk. In the presence of gas, the baryonic disk mass will be larger and so will be the halo contraction. The dark matter density contrast relative to the original elliptical will be therefore smaller.

Concluding, the differences between the baryon distributions of ellipticals and spirals are not sufficient to explain the overdensity of dark matter in ellipticals relative to spirals of the same luminosity or stellar mass. Ellipticals and spirals have not formed in the same halos. Instead, the higher dark matter density in ellipticals points to an earlier assembly redshift.

\section{THE DARK-HALO ASSEMBLY EPOCH OF COMA EARLY-TYPE GALAXIES}

In order to evaluate the difference between elliptical and spiral galaxy assembly redshifts quantitatively, let us assume that dark matter densities scale with the mean density of the universe at the assembly epoch, i.e., $\rho_{\mathrm{DM}} \propto\left(1+z_{\text {form }}\right)^{3}$ (we will discuss this assumption in Section 7.2). Let $z_{\text {form }}^{\mathrm{E}}$ and $z_{\text {form }}^{\mathrm{S}}$ denote the formation redshifts of ellipticals and spirals, respectively, then

$$
\frac{1+z_{\text {form }}^{\mathrm{E}}\left(L_{B}\right)}{1+z_{\text {form }}^{\mathrm{S}}\left(L_{B}\right)}=\left(\frac{\rho_{\mathrm{DM}}^{\mathrm{E}}\left(L_{B}\right)}{\rho_{\mathrm{DM}}^{\mathrm{S}}\left(L_{B}\right)}\right)^{1 / 3}
$$

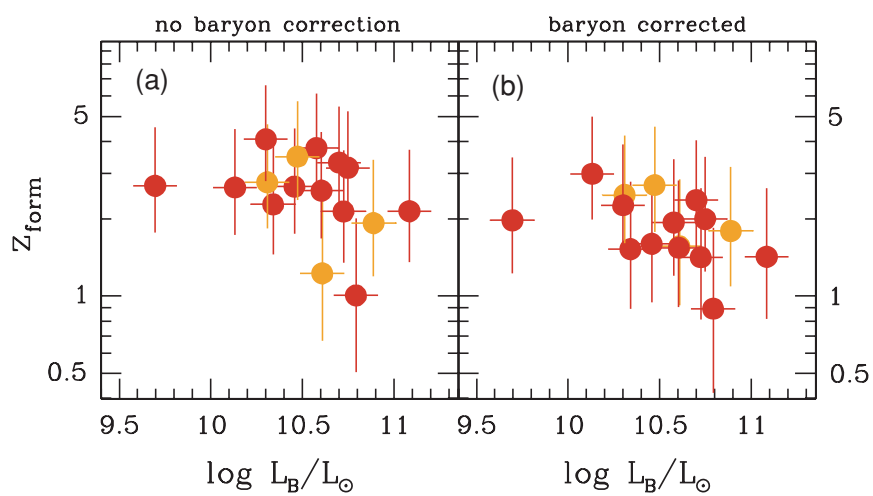

Figure 5. Estimated formation redshifts $z_{\text {form }}$ of Coma galaxy halos vs. luminosity without baryon correction $(a)$ and with baryon correction $(b)$. Symbols and colors as in Figure 1.

(A color version of this figure is available in the online journal.)

(Gerhard et al. 2001), with $\rho_{\mathrm{DM}}$ some measure of the dark matter density, e.g., $\rho_{\mathrm{DM}}=\left\langle\rho_{\mathrm{DM}}\right\rangle$. Equation (10) can be solved for

$$
z_{\text {form }}^{\mathrm{E}}=\left[1+z_{\text {form }}^{\mathrm{S}}\right] \times \delta^{1 / 3}-1
$$

where we have omitted the dependency of dark matter densities and formation redshifts on $L_{B}$ and defined $\delta=\rho_{\mathrm{DM}}^{\mathrm{E}} / \rho_{\mathrm{DM}}^{\mathrm{S}}$. Equation (11) allows to calculate formation redshifts of Coma ellipticals from $z_{\text {form }}^{\mathrm{S}}$ and the observed $\delta$. Two estimates based on different assumptions about $\delta$ and $z_{\text {form }}^{\mathrm{S}}$ are shown in Figure 5 and are discussed later. For each case, formation redshifts were calculated with both disk halo scaling laws shown in Figure 4 and the two results were averaged.

Raw formation redshifts without any baryon correction $(\delta=$ $\delta_{\text {obs }}$ ) are shown in Figure 5(a) (and listed in column 3 of Table 4). We considered a wide range of spiral galaxy formation redshifts 


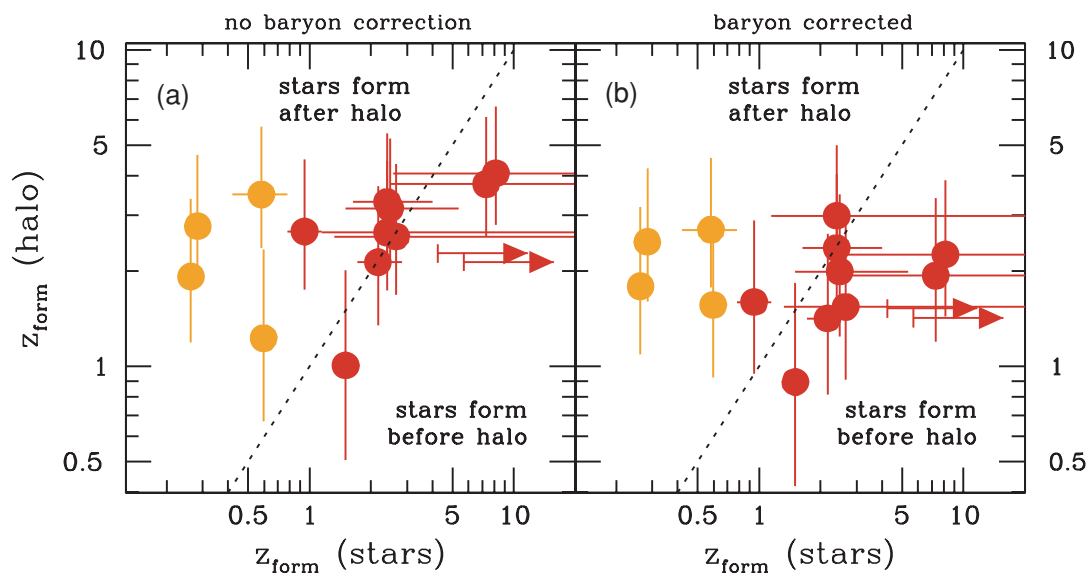

Figure 6. As Figure 5, but estimated formation redshifts $z_{\text {form }}$ of Coma galaxy halos are plotted vs. the formation epoch $z\left(\tau_{0}\right)$ of the central stellar population (from column 7 of Table 4); dotted lines: one-to-one relations. Symbols and colors as in Figure 1.

(A color version of this figure is available in the online journal.)

$z_{\text {form }}^{\mathrm{S}} \in[0.5,2]$ and the related uncertainty in $z_{\text {form }}^{\mathrm{E}}$ is indicated by vertical bars. Our fiducial value is $z_{\text {form }}^{\mathrm{S}} \equiv 1$, because regular disks become rare beyond $z \gtrsim 1$ (Conselice et al. 2005). We did not allow for a luminosity dependence of spiral galaxy formation times. For the Coma ellipticals, we then find $z_{\text {form }}^{\mathrm{E}}$ ranging from $z_{\text {form }}^{\mathrm{E}} \approx 0.5$ to $z_{\text {form }}^{\mathrm{E}} \approx 5$, with the majority of galaxies having formed around $z_{\text {form }}^{\mathrm{E}} \approx 3$. Brighter galaxies have assembled later than fainter galaxies.

Coma galaxy assembly redshifts shown in Figure 5(b) (see also column 5 of Table 4) include the baryon correction of Section 5, because we used $\delta=\delta_{\text {halo }}$ in Equation (11). Moreover, because fainter spirals have denser halos than brighter ones, we allowed for a luminosity dependent $z_{\text {form }}^{\mathrm{S}}(L)$. In analogy to Equation (10), assume

$$
\frac{1+z_{\text {form }}^{\mathrm{S}}(L)}{1+z_{\text {form }}^{\mathrm{S}}\left(L_{0}\right)}=\left(\frac{\rho_{\mathrm{DM}}^{\mathrm{S}}(L)}{\rho_{\mathrm{DM}}^{\mathrm{S}}\left(L_{0}\right)}\right)^{1 / 3}
$$

and $z_{0}^{\mathrm{S}}=1$ for a reference luminosity $\log L_{0} / L_{\odot}=10.5$. Because $\delta_{\text {halo }} \lesssim \delta_{\text {obs }}$, the baryon corrected $z_{\text {form }}^{\mathrm{E}}$ in Figure 5(b) are lower than the uncorrected ones in Figure 5(a). The typical assembly redshift reduces to $z_{\text {form }}^{\mathrm{E}} \approx 2$, as compared to $z_{\text {form }}^{\mathrm{E}} \approx 3$ without the correction. The baryon correction is mostly smaller than the uncertainty related to our ignorance about $z_{\text {form }}^{\mathrm{s}}$ (the vertical bars in Figure 5(b) correspond to $\left.z_{0}^{\mathrm{S}} \in[0.5,2]\right)$. The trend for lower $z_{\text {form }}^{\mathrm{E}}$ in brighter galaxies is slightly diminished by the baryon correction such that the dependency of $z_{\text {form }}^{\mathrm{E}}$ on $L$ in Figure 5(b) mainly reflects the luminosity dependence of spiral galaxy assembly redshifts.

Figure 6 compares halo assembly redshifts with central stellar population ages $\tau_{0}$ from Mehlert et al. (2003). We use $H_{0}=$ $70 \mathrm{~km} \mathrm{~s}^{-1} \mathrm{Mpc}^{-1}, \Omega_{\Lambda}=0.75$ and $\Omega_{m}=0.25$ to transform ages into redshifts. Largely independent from applying the baryon correction or not, the agreement between the two redshifts is fairly good for about half of our sample. Among the remaining galaxies, some have halos which appear younger than their central stellar populations. This could indicate that the stellar ages are overestimated (they are sometimes larger than the age of the universe in the adopted cosmology; see Table 4). It could also point at these galaxies having grown by dry merging. In a dry merger, the dark matter density can drop, but the stellar ages stay constant. In Coma galaxies with young stellar cores, the halo assembly redshifts are instead larger than the central stellar ages. This indicates some secondary star formation after the main epoch of halo assembly.

\section{COMPARISON WITH SEMI-ANALYTIC GALAXY FORMATION MODELS}

In the following we will compare our results to semi-analytic galaxy formation models. To this end we have constructed a comparison sample of synthetic ellipticals and spirals using the models of De Lucia \& Blaizot (2007), which are based on the Millennium simulation (Springel et al. 2005). Comparison ellipticals are selected to rest in dark matter cluster structures with virial masses larger than $M_{\mathrm{vir}}>10^{15} M_{\odot}$ and to obey $M_{B \text {,bulge }}-M_{B}<0.4$ (Simien \& De Vaucouleurs 1986). We ignore galaxies at the centers of simulated clusters since we have omitted the two central Coma galaxies from the analysis in this paper. Likewise, we exclude from the comparison galaxies that have been stripped-off their entire halo, because the only Coma galaxy that possibly lacks dark matter inside $3 r_{\text {eff }}$ has been excluded from the analysis in this paper as well (see Section 2). The isolated field spirals are drawn from objects with $M_{B, \text { bulge }}-M_{B}>1.56$ in the semi-analytic models (Simien \& De Vaucouleurs 1986).

Simulated galaxies were chosen randomly from the catalog of De Lucia \& Blaizot (2007) in a way such that each of six luminosity intervals (between $M_{B}=-17$ and $M_{B}=-23$; width $\Delta M_{B}=1.0$ ) contains roughly 50 galaxies. We use dustcorrected luminosities $M_{B}$ of the semi-analytic models.

\subsection{Dark Matter Density}

Dark matter halos of simulated galaxies are reconstructed from tabulated virial velocities $v_{\text {vir }}$, virial radii $r_{\text {vir }}$, and maximum circular velocities $v_{\max }$ as follows. It is assumed that the halos can be approximated by an NFW profile (see Equation (3)), in which case the circular velocity profile reads

$$
\left(\frac{v_{\mathrm{circ}}(r)}{v_{\mathrm{vir}}}\right)^{2}=\frac{1}{x} \frac{\ln (1+c x)-c x /(1+c x)}{\ln (1+c)-c /(1+c)} .
$$

Here, $x=r / r_{\text {vir }}$ and the halo concentration is defined by $c=r_{\mathrm{vir}} / r_{s}$. The maximum circular velocity $v_{\max }$ of an NFW halo occurs at $r \approx 2 r_{\text {vir }} / c$ (Navarro et al. 1996), such that (with Equation (13)) 


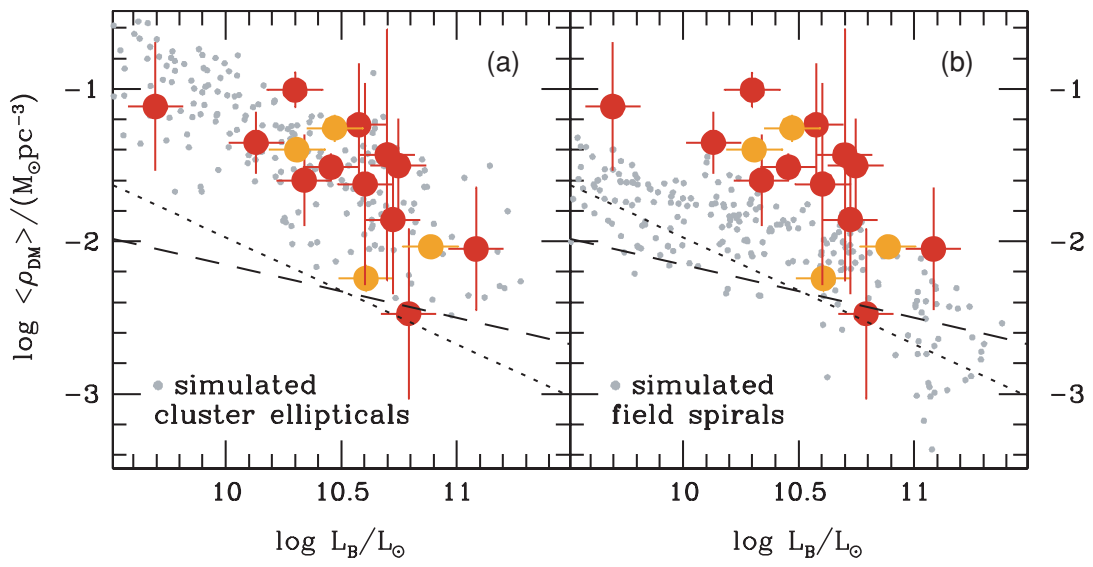

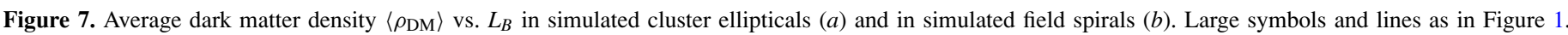
Simulated galaxies from the semi-analytic models of De Lucia \& Blaizot (2007).

(A color version of this figure is available in the online journal.)

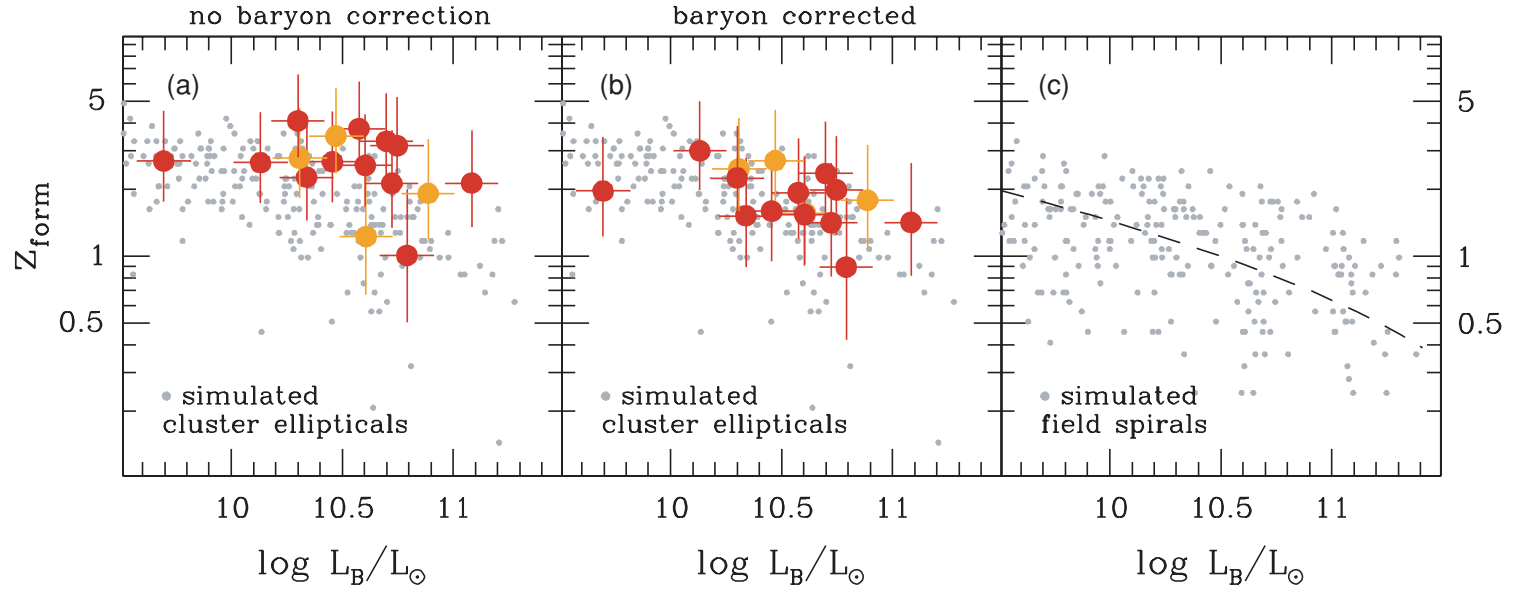

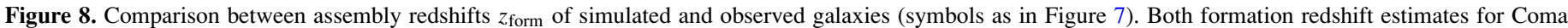

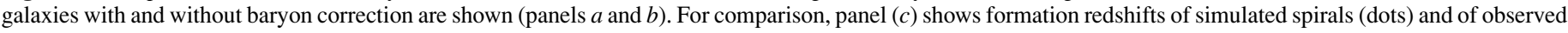
spirals (dashed line; see Equation (12)). Simulated galaxies from the semi-analytic models of De Lucia \& Blaizot (2007).

(A color version of this figure is available in the online journal.)

$$
4.63\left(\frac{v_{\max }}{v_{\text {vir }}}\right)^{2}=\frac{c}{\ln (1+c)-c /(1+c)} .
$$

Using the tabulated $v_{\text {vir }}$ and $v_{\max }$ this equation can be numerically solved for the halo concentration $c$, which in turn determines $r_{s}=r_{\mathrm{vir}} / c$ and, thus, the entire NFW profile of the halo.

Before compared to the Coma galaxy models, halo densities are averaged within $2 r_{\text {eff }}$ (see Equation (6)). In case of simulated spirals, we use effective radii from the empirical relation (4). For ellipticals, we assume

$$
\left(\frac{r_{\mathrm{eff}}}{\mathrm{kpc}}\right)=15.34\left(\frac{L_{B}}{10^{11} L_{\odot}}\right)^{1.02},
$$

which is a fit to the Coma data.

Figure 7 shows that the average dark matter densities $\left\langle\rho_{\mathrm{DM}}\right\rangle$ of the Coma early-type galaxies match fairly well with semianalytical models. This is remarkable, because the simulations do not take into account the halo response during baryon infall. Therefore, either the net effect of the baryons on the dark matter distribution is small in the analyzed population of galaxies or there is actually a mismatch between the halos of observed galaxies and the $N$-body models. It may also be that real galaxies do not have maximum stellar masses. This can be checked by the comparison of dynamically derived stellar mass-to-light ratios with independent stellar population synthesis models (J. Thomas et al. 2009, in preparation).

In semi-analytic models, similarly as in the case of real galaxies, dark matter densities are lower in spirals than in ellipticals (see Figure 7), but the density contrast in observed galaxies is larger. Again, a major uncertainty here is that the simulations do not take into account the gravitational effect of the baryons.

\subsection{Assembly Redshift}

Formation redshifts of simulated and observed galaxies are compared in Figure 8. Coma galaxy $z_{\text {form }}$ are from Section 6 and both cases discussed there-with and without baryon correction - are shown separately in Figures 8(a) and 8(b), respectively. The dashed line in Figure 8(c) shows the luminositydependent formation redshift $z_{\text {form }}^{\mathrm{S}}(L)$ of spiral galaxies that was assumed for the calculation of the baryon-corrected Coma galaxy $z_{\text {form }}$ (see Equation 12). Formation redshifts of simulated galaxies are defined as the earliest redshift, when a halo has assembled $50 \%$ of its maximum mass. Since we are mainly interested in cluster ellipticals, we need to take into account that interactions between the cluster halo and a galaxy's subhalo cause a mass loss in the latter. Although cluster-galaxy interactions happen in both simulated and observed galaxies, the mass 


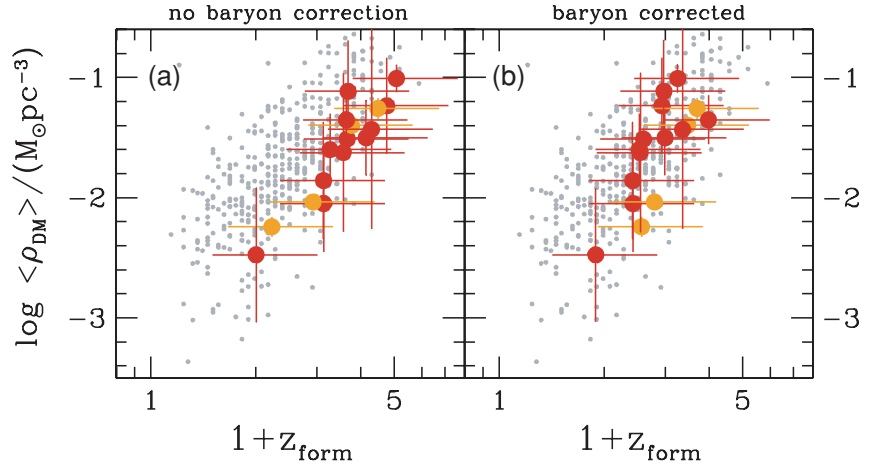

Figure 9. Average halo density $\left\langle\rho_{\mathrm{DM}}\right\rangle$ vs. assembly redshift. Large symbols: Coma galaxies without baryon correction $(a)$ and with baryon correction $(b)$. Small symbols: simulated cluster ellipticals and simulated field spirals from the semi-analytic models of De Lucia \& Blaizot (2007).

(A color version of this figure is available in the online journal.)

loss in the simulations may be overestimated because of the finite-numerical resolution and the neglect of the baryon potential. In particular, for simulated subhalos with very low masses at $z=0$, the derived formation redshifts may be artificially high, when defined according to the assembly of half of the final mass. To avoid such artificially large assembly redshifts, we define $z_{\text {form }}$ of simulated galaxies as the earliest time when half of the maximum mass was assembled, that a single progenitor in the merger tree of given galaxy had at some redshift. Our assumption is that even if dynamical interactions between cluster and galaxy halos take place, they do not significantly affect the very inner regions $<2 r_{\text {eff }}$ of interest here. In case of field spirals, formation redshifts defined either from the final or from the maximum mass are very similar.

Without a baryon correction, our estimates of Coma galaxy formation redshifts are on average higher than in the semianalytic models (Figure 8(a)). This, although (1) the dark matter densities of ellipticals match with the simulations and (2) our assumption about the formation redshifts of spirals $\left(z_{\text {form }}^{\mathrm{S}} \approx 1\right)$ is consistent with the simulations. The origin for the offset between Coma galaxies and semi-analytic models in Figure 8(a) is that the density contrast between halos of ellipticals and spirals is larger in observed galaxies than in the simulations. After applying the baryon correction, the Coma galaxy formation redshifts become consistent with the simulations (Figure 8(b)). This result indicates that the discrepancy between the measured and the simulated density ratio $\rho_{\mathrm{DM}}^{\mathrm{E}} / \rho_{\mathrm{DM}}^{\mathrm{S}}$ is due to baryon effects.

Our Coma galaxy formation redshifts are based on the assumption that $\left\langle\rho_{\mathrm{DM}}\right\rangle \propto\left(1+z_{\text {form }}\right)^{3}$. Figure 9 shows $\left\langle\rho_{\mathrm{DM}}\right\rangle$ versus $\left(1+z_{\text {form }}\right)$ explicitly. Independent of including a baryon correction or not, the slope of the relationship between $\left\langle\rho_{\mathrm{DM}}\right\rangle$ and $\left(1+z_{\text {form }}\right)$ in the Coma galaxies is roughly parallel to simulated $N$-body halos. This confirms that our assumption for the scaling between $\left\langle\rho_{\mathrm{DM}}\right\rangle$ and $z_{\text {form }}$ is approximately consistent with the cosmological simulations.

Concerning the absolute values of the dark matter densities, it has been stated earlier that they are only consistent with the simulations if either the net effect of the baryons is zero in the case of ellipticals or if galaxies do not have maximum stellar masses. The former case would imply that halos of spiral galaxies experience a net expansion during the baryon infall (several processes have been proposed for this, e.g., Binney et al. 2001).

De Lucia et al. (2006) quote a stellar assembly redshift below $z<1$ for simulated ellipticals more massive than
$M_{*}>10^{11} M_{\odot}$. The halo assembly redshifts in Figure 8 are mostly above $z>1$. In part, this is due to the fact that we only consider semi-analytic galaxies in high-density environments similar to Coma. In addition, formation redshifts defined according to the stellar mass assembly and the halo assembly, respectively, are not always equal. For example, in our comparison sample of simulated cluster ellipticals, we find an average dark halo assembly redshift $\left\langle z_{\text {form }}\right\rangle=1.50$ for galaxies more massive than $M_{*}>10^{11} M_{\odot}$. Evaluating for the same galaxies, the redshift $z_{\text {form }}^{*}$ (when half the stellar mass is assembled) yields $\left\langle z_{\text {form }}^{*}\right\rangle=1.07$. That $z_{\text {form }} \gtrsim z_{\text {form }}^{*}$ is plausible if some star formation is going on between $0 \leqslant z \leqslant z_{\text {form }}$ in the progenitor and/or in the subunits that are to be accreted after $z_{\text {form }}$. It should also be noted that the simulations do not take into account stellar mass loss due to tidal interactions.

\section{SUMMARY}

We have presented dark matter scaling relations derived from axisymmetric, orbit-based dynamical models of flattened and rotating as well as nonrotating Coma early-type galaxies. Dark matter halos in these galaxies follow similar trends with luminosity as for spirals. Thereby, the majority of Coma earlytype galaxies - those with old stellar populations-have halo core radii $r_{h}$ similar to spirals with the same $B$-band luminosity, but about 2.4 times higher asymptotic halo velocities. In contrast, four Coma early-type galaxies-with young central stellar populations - have halo velocities of the same order as in comparably bright spirals, but their core radii are smaller by a factor of 4. Differences between spirals and ellipticals increase when the comparison is made at the same stellar mass. The average halo density inside $2 r_{\text {eff }}$ exceeds that of comparably bright spirals by about a factor of 6.8. If the higher baryon concentration in ellipticals is taken into account, the excess density reduces to about a factor of 3 , but if ellipticals and spirals are compared at the same stellar mass, then it is again on the order of 6.5 .

Our measured dark matter densities match with a comparison sample of simulated cluster ellipticals constructed from the semi-analytic galaxy formation models of De Lucia \& Blaizot (2007). These synthetic ellipticals have $z_{\text {form }} \approx 0.5-4$ and higher dark matter densities than simulated field spirals, which appear on average around $z_{\text {form }}^{\mathrm{S}} \approx 1$.

Assuming for local spirals $z_{\text {form }}^{\mathrm{S}}=1$ as well, and assuming further that the inner dark matter density scales with the formation redshift like $\left(1+z_{\text {form }}\right)^{3}$, our results imply that ellipticals have formed $\Delta z_{\text {form }} \approx 1-2$ earlier than spirals. Without baryon correction, we find an average formation redshift around $z_{\text {form }} \approx 3$, which is slightly larger than in semi-analytic galaxy formation models. Accounting for the more concentrated baryons in ellipticals, the average formation redshift drops to $z_{\text {form }} \approx 2$.

For about half of our sample, dark halo formation redshifts match with constraints derived from stellar populations (Mehlert et al. 2003): the assembly epoch of these (old) early-type galaxies coincides with the epoch of formation of their stellar components.

We thank Ortwin Gerhard and the anonymous referee for comments and suggestions that helped to improve the manuscript. J.T. acknowledges financial support by the Sonderforschungsbereich 375 "Astro-Teilchenphysik" of the Deutsche Forschungsgemeinschaft. EMC receives support from grant CPDA068415/06 by Padua University. The Millennium Sim- 
GMP3414

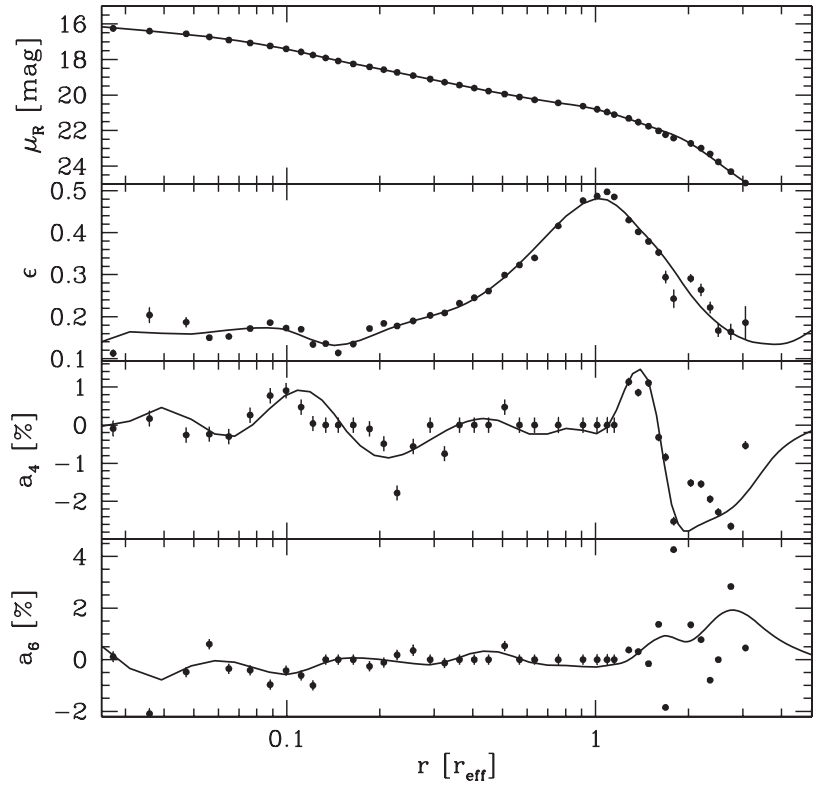

GMP3414

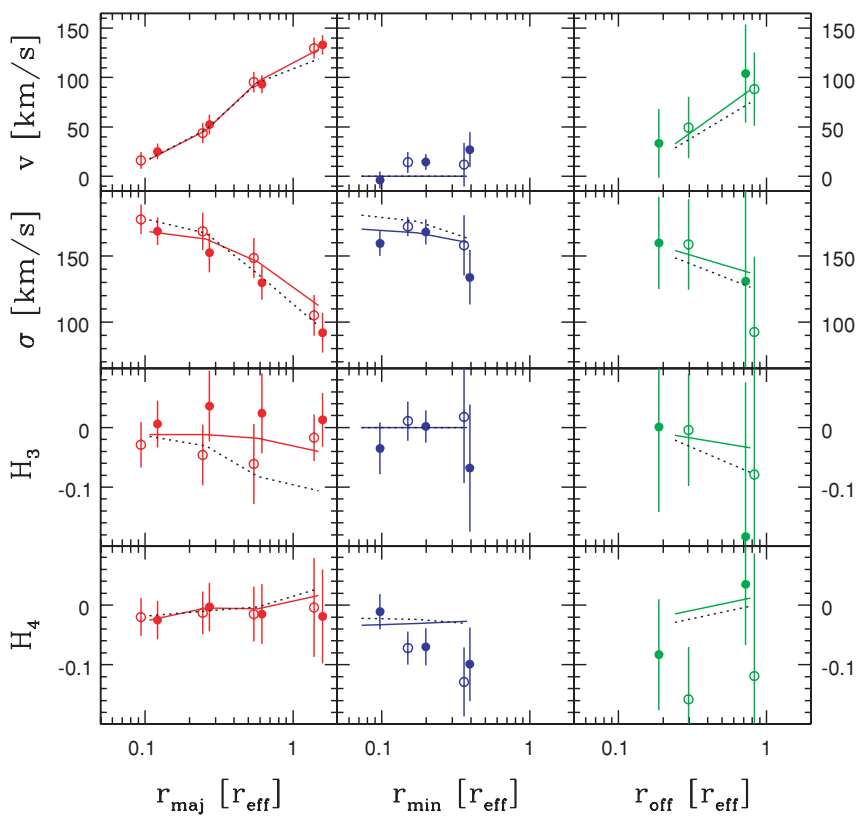

Figure 10. Upper panel: joint ground based and HST photometry of GMP 3414/ NGC 4871. Lines: best-fit deprojection. Lower panel: stellar kinematics along major axis (left/red), along the minor axis (middle/blue), and along a third axis parallel to the major axis with an offset of $r_{\text {eff }} / 2$ (right/green); filled and open circles refer to different sides of the slits; dotted: best-fit model without dark matter.

(A color version of this figure is available in the online journal.)

ulation databases used in this paper and the web application providing online access to them were constructed as part of the activities of the German Astrophysical Virtual Observatory.

\section{APPENDIX}

\section{GMP 3414 AND GMP 4822}

The best-fit model parameters for the galaxies GMP 3414 and GMP 4822 (which were not included in the original sample of Thomas et al. 2007a) are given in Table 1. The table is similar to Table 2 of Thomas et al. (2007a) and we refer the reader to this
GMP4822

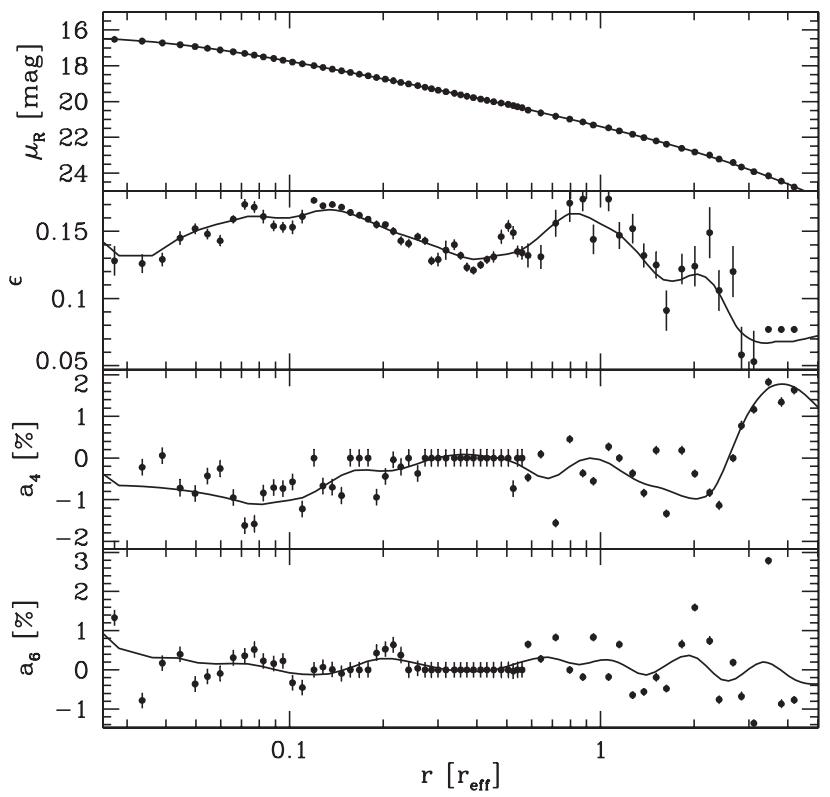

GMP4822

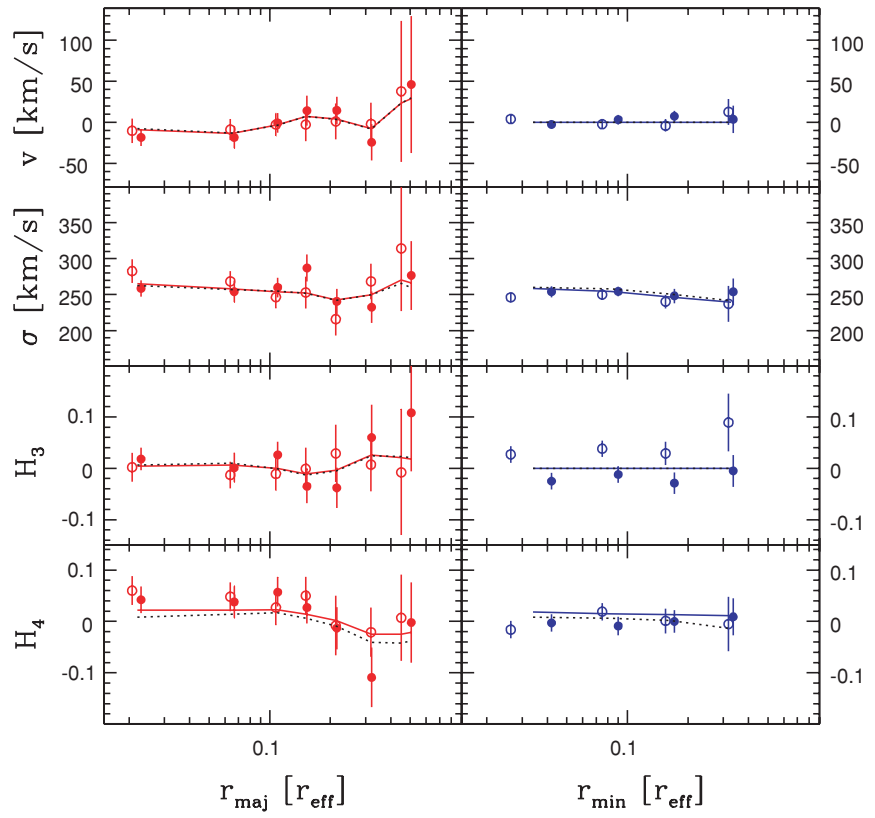

Figure 11. Same as Figure 10 but for GMP 4822/NGC 4841A.

(A color version of this figure is available in the online journal.)

paper, in case more detailed information about the parameter definitions are required. The best-fit models with and without dark matter halo are compared to the observations in Figures 10 and 11. In both galaxies, the best-fit inclination is $i=90^{\circ}$, but the $68 \%$ confidence regions include models at $i \geqslant 70^{\circ}$ (GMP 3414) and $i \geqslant 50^{\circ}$ (GMP 4822).

GMP 3414 and GMP 4822 were observed with the Wide Field Planetary Camera 2 (WFPC2) on board the HST as part of the HST proposal 10844 (PI: G. Wegner). For each galaxy two exposures with 300 s each were taken with the filter F622W. Four other objects were previously observed as part of this proposal and a full description of the respective observational parameters and the data analysis is given in Corsini et al. (2008). In Table 5 we just list the final photometric parameters. 
Table 5

Photometric Parameters of the Galaxies GMP 3414 and GMP 4822 Observed with HST/WFPC2

\begin{tabular}{|c|c|c|c|c|c|c|c|c|c|c|c|c|c|}
\hline $\begin{array}{l}a \\
(\operatorname{arcsec})\end{array}$ & $\begin{array}{c}\mu_{R} \\
\left(\mathrm{mag} \mathrm{arcsec}^{-2}\right)\end{array}$ & $e$ & $\begin{array}{l}\text { P.A. } \\
\left({ }^{\circ}\right)\end{array}$ & $\begin{array}{c}\Delta x_{\mathrm{c}} \\
(\operatorname{arcsec})\end{array}$ & $\begin{array}{c}\Delta y_{\mathrm{c}} \\
(\operatorname{arcsec})\end{array}$ & $\begin{array}{c}\text { Err. }^{\mathrm{a}} \\
(\operatorname{arcsec})\end{array}$ & $\begin{array}{l}a_{3} / a \\
\times 100\end{array}$ & $\begin{array}{l}b_{3} / a \\
\times 100\end{array}$ & $\begin{array}{l}a_{4} / a \\
\times 100\end{array}$ & $\begin{array}{l}b_{4} / a \\
\times 100\end{array}$ & $\begin{array}{l}a_{6} / a \\
\times 100\end{array}$ & $\begin{array}{l}b_{6} / a \\
\times 100\end{array}$ & Err. $^{b}$ \\
\hline \multicolumn{14}{|c|}{ GMP 3414} \\
\hline $0.016 \pm 0.004$ & $14.791 \pm 0.015$ & $0.134 \pm 0.284$ & $161.3 \pm 70.1$ & 0.000 & 0.000 & 0.003 & -0.440 & 2.470 & -4.720 & -1.980 & -0.600 & -0.050 & 1.148 \\
\hline $0.049 \pm 0.004$ & $15.018 \pm 0.015$ & $0.152 \pm 0.095$ & $160.3 \pm 21.2$ & 0.003 & 0.001 & 0.003 & -0.560 & 2.920 & -6.630 & -3.640 & -0.430 & -0.720 & 0.991 \\
\hline $0.070 \pm 0.001$ & $15.271 \pm 0.012$ & $0.109 \pm 0.023$ & $162.0 \pm 6.9$ & 0.005 & 0.000 & 0.001 & 0.640 & 0.480 & -2.990 & -1.480 & 0.180 & -0.490 & 0.215 \\
\hline $0.088 \pm 0.001$ & $15.404 \pm 0.016$ & $0.097 \pm 0.011$ & $162.4 \pm 3.5$ & 0.007 & -0.001 & 0.001 & 0.930 & -0.440 & -1.270 & -0.800 & 0.290 & -0.640 & 0.219 \\
\hline $0.108 \pm 0.001$ & $15.562 \pm 0.015$ & $0.139 \pm 0.010$ & $162.0 \pm 2.3$ & 0.010 & -0.002 & 0.001 & 1.470 & -0.620 & -0.330 & -0.530 & 0.230 & -1.000 & 0.311 \\
\hline $0.132 \pm 0.001$ & $15.725 \pm 0.011$ & $0.117 \pm 0.007$ & $160.6 \pm 1.9$ & 0.013 & -0.003 & 0.001 & 0.520 & -0.300 & -0.510 & 0.480 & 1.050 & -0.720 & 0.246 \\
\hline $0.156 \pm 0.001$ & $15.886 \pm 0.010$ & $0.128 \pm 0.007$ & $158.3 \pm 1.9$ & 0.016 & -0.002 & 0.001 & 0.420 & -0.350 & 0.030 & 0.520 & 1.380 & -0.740 & 0.371 \\
\hline $0.189 \pm 0.001$ & $16.068 \pm 0.010$ & $0.132 \pm 0.008$ & $157.7 \pm 2.0$ & 0.025 & 0.000 & 0.001 & 1.110 & -1.060 & 0.380 & -0.610 & 1.370 & -0.930 & 0.280 \\
\hline $0.228 \pm 0.002$ & $16.248 \pm 0.009$ & $0.113 \pm 0.008$ & $152.3 \pm 2.4$ & 0.042 & 0.005 & 0.001 & 1.250 & -1.320 & -0.090 & -0.770 & 0.120 & 0.970 & 0.415 \\
\hline $0.299 \pm 0.005$ & $16.399 \pm 0.007$ & $0.204 \pm 0.018$ & $141.9 \pm 3.2$ & 0.044 & 0.003 & 0.003 & 2.110 & 4.250 & 0.170 & -2.910 & -2.090 & -0.920 & 1.566 \\
\hline $0.393 \pm 0.004$ & $16.553 \pm 0.005$ & $0.187 \pm 0.012$ & $126.8 \pm 2.3$ & 0.004 & -0.006 & 0.003 & 1.300 & 3.840 & -0.260 & 2.370 & -0.480 & -1.110 & 0.592 \\
\hline $0.467 \pm 0.002$ & $16.730 \pm 0.005$ & $0.150 \pm 0.005$ & $141.6 \pm 1.2$ & -0.010 & -0.002 & 0.002 & 1.080 & 0.630 & -0.240 & -0.620 & 0.600 & 0.180 & 0.505 \\
\hline $0.540 \pm 0.002$ & $16.905 \pm 0.005$ & $0.153 \pm 0.005$ & $141.9 \pm 1.2$ & -0.012 & 0.002 & 0.002 & 0.990 & 0.320 & -0.300 & -0.890 & -0.350 & 0.210 & 0.563 \\
\hline $0.633 \pm 0.003$ & $17.068 \pm 0.005$ & $0.172 \pm 0.005$ & $139.2 \pm 1.0$ & -0.016 & -0.002 & 0.002 & -0.280 & 1.020 & 0.260 & -1.470 & -0.410 & 0.300 & 0.496 \\
\hline $0.735 \pm 0.004$ & $17.234 \pm 0.004$ & $0.186 \pm 0.006$ & $135.4 \pm 1.2$ & -0.021 & 0.004 & 0.003 & -0.530 & 0.380 & 0.770 & -2.930 & -0.970 & -0.580 & 0.421 \\
\hline $0.828 \pm 0.004$ & $17.404 \pm 0.005$ & $0.173 \pm 0.005$ & $134.9 \pm 1.0$ & -0.019 & -0.003 & 0.003 & -0.270 & 0.200 & 0.900 & -2.020 & -0.430 & -1.210 & 0.296 \\
\hline $0.926 \pm 0.004$ & $17.577 \pm 0.005$ & $0.170 \pm 0.005$ & $136.8 \pm 1.0$ & -0.016 & 0.000 & 0.003 & -0.510 & 0.150 & 0.470 & -0.870 & -0.610 & -0.340 & 0.448 \\
\hline $1.011 \pm 0.004$ & $17.747 \pm 0.005$ & $0.134 \pm 0.005$ & $141.2 \pm 1.2$ & -0.014 & 0.006 & 0.003 & -0.230 & 0.010 & 0.040 & -0.900 & -1.000 & -0.020 & 0.387 \\
\hline $1.112 \pm 0.004$ & $17.914 \pm 0.005$ & $0.136 \pm 0.005$ & $147.7 \pm 1.1$ & -0.016 & 0.007 & 0.003 & $\ldots$ & $\ldots$ & $\ldots$ & & & $\ldots$ & $\ldots$ \\
\hline $1.221 \pm 0.005$ & $18.082 \pm 0.004$ & $0.114 \pm 0.005$ & $155.1 \pm 1.5$ & -0.007 & 0.009 & 0.004 & & $\ldots$ & & & & & \\
\hline $1.366 \pm 0.006$ & $18.250 \pm 0.004$ & $0.135 \pm 0.005$ & $160.7 \pm 1.3$ & -0.017 & 0.013 & 0.004 & $\ldots$ & $\ldots$ & $\ldots$ & $\ldots$ & $\ldots$ & $\ldots$ & $\ldots$ \\
\hline $1.542 \pm 0.007$ & $18.418 \pm 0.004$ & $0.172 \pm 0.006$ & $163.7 \pm 1.1$ & -0.015 & 0.014 & 0.005 & -0.400 & -0.310 & -0.100 & 0.070 & -0.260 & 1.370 & 0.468 \\
\hline $1.715 \pm 0.009$ & $18.573 \pm 0.004$ & $0.184 \pm 0.006$ & $166.5 \pm 1.1$ & -0.009 & 0.008 & 0.006 & -0.380 & -0.040 & -0.490 & 1.360 & -0.110 & 0.260 & 0.466 \\
\hline $1.894 \pm 0.011$ & $18.726 \pm 0.004$ & $0.178 \pm 0.007$ & $166.6 \pm 1.3$ & 0.015 & 0.037 & 0.008 & 0.270 & -0.710 & -1.780 & -0.200 & 0.180 & 0.100 & 0.567 \\
\hline $2.134 \pm 0.012$ & $18.906 \pm 0.003$ & $0.190 \pm 0.006$ & $166.8 \pm 1.2$ & -0.007 & 0.017 & 0.008 & 0.020 & -0.720 & -0.560 & 0.230 & 0.350 & -0.270 & 0.615 \\
\hline $2.424 \pm 0.009$ & $19.103 \pm 0.003$ & $0.203 \pm 0.004$ & $169.4 \pm 0.7$ & -0.035 & 0.034 & 0.006 & $\ldots$ & $\ldots$ & $\ldots$ & $\ldots$ & $\ldots$ & $\ldots$ & $\ldots$ \\
\hline $2.699 \pm 0.018$ & $19.279 \pm 0.004$ & $0.209 \pm 0.007$ & $171.0 \pm 1.3$ & -0.031 & 0.052 & 0.013 & 0.220 & 0.120 & -0.750 & 1.650 & -0.130 & 0.180 & 0.538 \\
\hline $3.014 \pm 0.015$ & $19.443 \pm 0.004$ & $0.232 \pm 0.006$ & $171.2 \pm 0.9$ & -0.042 & 0.022 & 0.011 & $\ldots$ & $\ldots$ & $\ldots$ & $\ldots$ & $\ldots$ & $\ldots$ & $\ldots$ \\
\hline $3.367 \pm 0.013$ & $19.611 \pm 0.004$ & $0.245 \pm 0.004$ & $172.7 \pm 0.7$ & -0.024 & -0.007 & 0.009 & $\ldots$ & $\ldots$ & $\cdots$ & $\cdots$ & $\ldots$ & $\ldots$ & $\ldots$ \\
\hline $3.745 \pm 0.016$ & $19.780 \pm 0.004$ & $0.261 \pm 0.004$ & $175.0 \pm 0.7$ & -0.010 & 0.021 & 0.011 & $\ldots$ & $\ldots$ & $\ldots$ & $\ldots$ & $\ldots$ & $\ldots$ & $\ldots$ \\
\hline $4.222 \pm 0.020$ & $19.950 \pm 0.004$ & $0.299 \pm 0.005$ & $174.9 \pm 0.6$ & -0.004 & 0.088 & 0.014 & -0.120 & 0.530 & 0.470 & 0.110 & 0.530 & -0.400 & 0.448 \\
\hline $4.716 \pm 0.020$ & $20.114 \pm 0.004$ & $0.323 \pm 0.004$ & $175.3 \pm 0.5$ & -0.015 & 0.047 & 0.014 & $\ldots$ & $\ldots$ & $\ldots$ & $\ldots$ & & & \\
\hline $5.280 \pm 0.039$ & $20.272 \pm 0.004$ & $0.340 \pm 0.007$ & $176.1 \pm 0.9$ & -0.019 & 0.040 & 0.028 & $\ldots$ & $\ldots$ & $\ldots$ & & & & \\
\hline $6.295 \pm 0.036$ & $20.440 \pm 0.004$ & $0.416 \pm 0.005$ & $176.7 \pm 0.6$ & -0.046 & 0.084 & 0.026 & $\ldots$ & $\ldots$ & $\ldots$ & $\ldots$ & $\ldots$ & & \\
\hline $7.573 \pm 0.048$ & $20.626 \pm 0.004$ & $0.476 \pm 0.005$ & $176.6 \pm 0.5$ & -0.073 & 0.182 & 0.034 & & . & & & & . & \\
\hline $8.416 \pm 0.063$ & $20.805 \pm 0.005$ & $0.487 \pm 0.005$ & $177.2 \pm 0.6$ & -0.065 & 0.100 & 0.045 & $\ldots$ & $\ldots$ & $\ldots$ & . & . & . & $\ldots$ \\
\hline $9.065 \pm 0.057$ & $20.957 \pm 0.006$ & $0.497 \pm 0.004$ & $177.5 \pm 0.5$ & -0.065 & 0.051 & 0.040 & $\cdots$ & $\cdots$ & & & & & \\
\hline $9.560 \pm 0.065$ & $21.102 \pm 0.006$ & $0.485 \pm 0.005$ & $177.4 \pm 0.6$ & -0.072 & 0.242 & 0.046 & $\ldots$ & . & & & & & \\
\hline $10.087 \pm 0.122$ & $21.228 \pm 0.007$ & $0.483 \pm 0.009$ & $177.6 \pm 1.0$ & -0.115 & 0.121 & 0.086 & & $\ldots$ & & & & & \\
\hline $10.584 \pm 0.105$ & $21.361 \pm 0.008$ & $0.475 \pm 0.007$ & $178.1 \pm 0.9$ & -0.078 & 0.077 & 0.074 & $\ldots$ & $\ldots$ & & & & & \\
\hline $10.912 \pm 0.126$ & $21.502 \pm 0.010$ & $0.458 \pm 0.009$ & $177.1 \pm 1.0$ & -0.053 & 0.072 & 0.089 & & $\ldots$ & & & $\ldots$ & $\ldots$ & \\
\hline $11.361 \pm 0.226$ & $21.616 \pm 0.010$ & $0.464 \pm 0.015$ & $177.6 \pm 1.7$ & -0.133 & 0.131 & 0.160 & $\ldots$ & $\ldots$ & $\ldots$ & $\ldots$ & $\ldots$ & $\ldots$ & $\ldots$ \\
\hline
\end{tabular}

GMP 4822

$0.373 \pm 0.006 \quad 16.419 \pm 0.003 \quad 0.163 \pm 0.019 \quad 116.2 \pm 4.0$ $0.489 \pm 0.00516 .526 \pm 0.003 \quad 0.128 \pm 0.011 \quad 111.5 \pm 2.9$ $0.600 \pm 0.00316 .623 \pm 0.003 \quad 0.126 \pm 0.007 \quad 115.9 \pm 1.7$ $0.697 \pm 0.003 \quad 16.726 \pm 0.003 \quad 0.129 \pm 0.005116 .4 \pm 1.3$ $0.795 \pm 0.003 \quad 16.827 \pm 0.003 \quad 0.145 \pm 0.005116 .6 \pm 1.1$ $0.888 \pm 0.00316 .925 \pm 0.003 \quad 0.152 \pm 0.004113 .8 \pm 0.9$ $0.973 \pm 0.00317 .023 \pm 0.003 \quad 0.148 \pm 0.004112 .8 \pm 1.0$ $1.069 \pm 0.00417 .120 \pm 0.003 \quad 0.143 \pm 0.004112 .1 \pm 0.9$ $1.178 \pm 0.003 \quad 17.219 \pm 0.003 \quad 0.159 \pm 0.003 \quad 111.3 \pm 0.7$ $1.282 \pm 0.00517 .310 \pm 0.004 \quad 0.170 \pm 0.004 \quad 112.0 \pm 0.9$ $\begin{array}{lllll}1.376 & \pm 0.005 & 17.406 \pm 0.004 & 0.168 \pm 0.004 & 111.3 \pm 0.9\end{array}$ $1.472 \pm 0.00617 .505 \pm 0.003 \quad 0.161 \pm 0.005110 .7 \pm 1.0$ $1.589 \pm 0.00617 .593 \pm 0.007 \quad 0.154 \pm 0.004112 .0 \pm 1.0$ $1.702 \pm 0.00617 .693 \pm 0.007 \quad 0.153 \pm 0.004111 .1 \pm 0.9$ $1.826 \pm 0.007 \quad 17.794 \pm 0.003 \quad 0.153 \pm 0.005110 .4 \pm 1.0$ $1.964 \pm 0.00917 .891 \pm 0.002 \quad 0.161 \pm 0.005 \quad 109.7 \pm 1.1$ $2.141 \pm 0.004 \quad 17.993 \pm 0.002 \quad 0.173 \pm 0.002 \quad 108.7 \pm 0.5$ $2.288 \pm 0.00318 .095 \pm 0.0020 .169 \pm 0.002 \quad 109.4 \pm 0.4$ $2.452 \pm 0.004 \quad 18.192 \pm 0.002 \quad 0.170 \pm 0.002 \quad 109.6 \pm 0.4$ $2.626 \pm 0.00518 .282 \pm 0.0020 .168 \pm 0.002108 .9 \pm 0.5$

$\begin{array}{rrrrrrrrrr}-0.007 & -0.000 & 0.004 & -1.560 & 3.930 & 0.140 & 0.700 & 2.850 & 3.670 & 1.309 \\ -0.014 & 0.008 & 0.003 & -0.310 & 1.830 & -2.180 & 0.420 & 1.330 & -0.650 & 0.973 \\ -0.018 & 0.007 & 0.002 & -0.840 & 0.180 & -0.220 & -0.160 & -0.780 & 0.270 & 0.666 \\ -0.018 & 0.012 & 0.002 & 0.870 & -0.150 & 0.060 & -0.100 & 0.170 & -0.270 & 0.556 \\ -0.020 & 0.006 & 0.002 & 0.170 & -1.160 & -0.720 & 0.590 & 0.400 & 0.800 & 0.474 \\ -0.017 & 0.010 & 0.002 & -0.090 & -0.280 & -0.850 & 0.560 & -0.360 & 0.940 & 0.390 \\ -0.020 & 0.012 & 0.002 & 0.270 & -0.320 & -0.430 & 0.160 & -0.170 & 0.160 & 0.395 \\ -0.012 & 0.007 & 0.002 & -0.150 & -0.400 & -0.250 & 0.520 & -0.090 & -0.150 & 0.309 \\ -0.008 & 0.008 & 0.002 & -0.190 & -0.520 & -0.950 & 0.250 & 0.310 & -0.470 & 0.299 \\ -0.017 & 0.014 & 0.003 & 0.050 & 0.040 & -1.620 & 0.920 & 0.360 & -0.000 & 0.330 \\ -0.017 & 0.015 & 0.003 & 0.130 & -0.050 & -1.580 & 0.910 & 0.520 & -0.130 & 0.356 \\ -0.018 & 0.006 & 0.004 & 0.630 & 0.160 & -0.840 & 0.190 & 0.230 & -0.600 & 0.532 \\ -0.015 & 0.004 & 0.004 & 0.010 & -0.240 & -0.710 & -0.190 & 0.160 & 0.370 & 0.317 \\ -0.028 & 0.007 & 0.004 & 0.230 & -0.000 & -0.730 & 0.210 & 0.230 & -0.080 & 0.377 \\ -0.026 & 0.003 & 0.005 & 0.150 & 0.280 & -0.570 & 0.240 & -0.330 & 0.140 & 0.479 \\ -0.028 & 0.019 & 0.006 & 0.060 & 0.020 & -1.220 & 0.260 & -0.450 & -0.130 & 0.374 \\ -0.015 & 0.010 & 0.003 & \ldots & \ldots & \ldots & \ldots & \ldots & \ldots & \ldots \\ -0.020 & 0.006 & 0.002 & 0.140 & -0.180 & -0.670 & 0.140 & 0.070 & -0.060 & 0.133 \\ -0.022 & 0.006 & 0.003 & 0.100 & -0.140 & -0.700 & 0.390 & 0.010 & -0.060 & 0.167 \\ -0.026 & 0.008 & 0.004 & -0.240 & 0.140 & -0.900 & 0.070 & -0.090 & 0.340 & 0.145\end{array}$


Table 5

(Continued)

\begin{tabular}{|c|c|c|c|c|c|c|c|c|c|c|c|c|c|}
\hline $\begin{array}{l}a \\
(\operatorname{arcsec})\end{array}$ & $\begin{array}{c}\mu_{R} \\
\left(\operatorname{mag~}^{\left.\operatorname{arcsec}^{-2}\right)}\right.\end{array}$ & $e$ & $\begin{array}{l}\text { P.A. } \\
\left({ }^{\circ}\right)\end{array}$ & $\begin{array}{c}\Delta x_{\mathrm{c}} \\
(\operatorname{arcsec})\end{array}$ & $\begin{array}{c}\Delta y_{\mathrm{c}} \\
(\operatorname{arcsec})\end{array}$ & $\begin{array}{c}\text { Err. }^{\mathrm{a}} \\
(\operatorname{arcsec})\end{array}$ & $\begin{array}{l}a_{3} / a \\
\times 100 \\
\end{array}$ & $\begin{array}{l}b_{3} / a \\
\times 100 \\
\end{array}$ & $\begin{array}{l}a_{4} / a \\
\times 100 \\
\end{array}$ & $\begin{array}{l}b_{4} / a \\
\times 100 \\
\end{array}$ & $\begin{array}{l}a_{6} / a \\
\times 100 \\
\end{array}$ & $\begin{array}{l}b_{6} / a \\
\times 100 \\
\end{array}$ & Err. $^{b}$ \\
\hline $2.803 \pm 0.005$ & $18.378 \pm 0.002$ & $0.164 \pm 0.002$ & $108.7 \pm 0.4$ & -0.023 & 0.002 & & & & & & & & \\
\hline $2.995 \pm 0.006$ & $18.472 \pm 0.002$ & $.162 \pm 0.002$ & $109.2 \pm 0.5$ & -0.016 & 0.007 & 0.005 & & & & & & & $\ldots$ \\
\hline $3.196 \pm 0.006$ & $18.563 \pm 0.004$ & $.159 \pm 0.002$ & $107.5 \pm 0.5$ & -0.021 & 0.008 & 0.004 & $\ldots$ & $\ldots$ & $\ldots$ & & & & $\ldots$ \\
\hline $3.402 \pm 0.008$ & $18.657 \pm 0.004$ & $155 \pm 0.003$ & $108.1 \pm 0.6$ & -0.026 & 0.003 & .000 & 0.120 & -0.130 & -0.940 & -0.030 & & 0.120 & 0.249 \\
\hline $3.631 \pm 0.007$ & $18.749 \pm 0.002$ & $155 \pm 0.002$ & $107.4 \pm 0.5$ & -0.027 & .001 & 005 & -0.290 & -0.080 & -0.440 & -0.070 & & 0.400 & 0.204 \\
\hline $3.854 \pm 0.008$ & $18.839 \pm 0.002$ & $0.150 \pm 0.003$ & $107.9 \pm 0.6$ & -0.023 & 0.009 & 0.006 & -0.240 & -0.050 & -0.040 & 0.080 & 0.640 & -0.060 & 0.268 \\
\hline $4.073 \pm 0.010$ & $18.932 \pm 0.002$ & $0.143 \pm 0.003$ & $108.2 \pm 0.7$ & -0.017 & 0.011 & 0.007 & -0.490 & 0.220 & -0.210 & 0.320 & 0.380 & -0.100 & 0.233 \\
\hline $4.314 \pm 0.012$ & $19.019 \pm 0.002$ & $0.141 \pm 0.003$ & $108.0 \pm 0.8$ & -0.009 & 0.013 & & $\ldots$ & $\ldots$ & $\ldots$ & $\ldots$ & & $\ldots$ & $\cdots$ \\
\hline $4.614 \pm 0.010$ & $19.110 \pm 0.002$ & $0.146 \pm 0.003$ & $106.2 \pm 0.6$ & 0.003 & 0.008 & 0.007 & -0.220 & 0.010 & -0.370 & -0.260 & 0.040 & -0.070 & 0.168 \\
\hline $4.872 \pm 0.013$ & $19.200 \pm 0.002$ & $0.143 \pm 0.003$ & $107.4 \pm 0.8$ & -0.016 & -0.018 & 09 & $\ldots$ & & & & & $\ldots$ & $\ldots$ \\
\hline $5.111 \pm 0.012$ & $19.283 \pm 0.003$ & $0.128 \pm 0.003$ & $109.0 \pm 0.7$ & -0.023 & 0.026 & 0.008 & $\ldots$ & $\cdots$ & $\ldots$ & $\cdots$ & $\ldots$ & $\cdots$ & $\ldots$ \\
\hline $5.369 \pm 0.020$ & $19.367 \pm 0.003$ & $0.129 \pm 0.005$ & $109.0 \pm 1.2$ & -0.011 & 0.040 & & $\cdots$ & $\cdots$ & $\cdots$ & & & & $\cdots$ \\
\hline $5.688 \pm 0.031$ & $19.453 \pm 0.003$ & $0.136 \pm 0.007$ & $108.7 \pm 1.6$ & 0.005 & & & & & $\cdots$ & & & & . \\
\hline $6.054 \pm 0.018$ & $19.541 \pm 0.003$ & $0.140 \pm 0.004$ & $108.8 \pm 0.9$ & 0.016 & 0.039 & & & & & & & & \\
\hline $6.343 \pm 0.017$ & $19.628 \pm 0.003$ & $0.132 \pm 0.003$ & $106.7 \pm 0.8$ & 0.030 & 0.041 & & & & & & & & \\
\hline $6.641 \pm 0.016$ & $19.705 \pm 0.003$ & $0.123 \pm 0.003$ & $106.4 \pm 0.8$ & 0.014 & 0.020 & 0.0 & & & $\ldots$ & $\cdots$ & & & $\ldots$ \\
\hline $6.970 \pm 0.019$ & $19.785 \pm 0.003$ & $0.121 \pm 0.003$ & $104.3 \pm 0.9$ & 0.023 & 0.006 & & & & $\cdots$ & & & $\cdots$ & $\cdots$ \\
\hline $7.353 \pm 0.017$ & $19.863 \pm 0.003$ & $0.125 \pm 0.003$ & $105.9 \pm 0.8$ & 0.032 & 0.033 & 0.012 & & & & & & & $\cdots$ \\
\hline $7.703 \pm 0.019$ & $19.936 \pm 0.003$ & $0.129 \pm 0.003$ & $104.1 \pm 0.8$ & 0.009 & & & & & & & & & \\
\hline $8.098 \pm 0.028$ & $20.011 \pm 0.003$ & $0.131 \pm 0.004$ & $104.4 \pm 1.1$ & & & & & & & & & & \\
\hline $8.559 \pm 0.033$ & $20.086 \pm 0.003$ & $0.146 \pm 0.005$ & $102.9 \pm 1.1$ & & -0.005 & & & & & & & $\cdots$ & \\
\hline $9.016 \pm 0.034$ & $20.159 \pm 0.004$ & $0.154 \pm 0.004$ & $102.0 \pm 1.0$ & 0.012 & -0.010 & & & $\ldots$ & $\ldots$ & $\ldots$ & $\ldots$ & $\ldots$ & $\ldots$ \\
\hline $9.363 \pm 0.035$ & $20.228 \pm 0.004$ & $0.149 \pm 0.005$ & $101.7 \pm 1.0$ & 0.027 & 0.006 & 0.025 & 0.260 & -0.380 & -0.730 & -0.040 & -0.030 & 0.050 & 0.347 \\
\hline $9.653 \pm 0.035$ & $20.290 \pm 0.004$ & $0.135 \pm 0.004$ & $103.3 \pm 1.1$ & 0.086 & 0.048 & 0.025 & $\ldots$ & $\ldots$ & $\ldots$ & $\ldots$ & $\ldots$ & $\ldots$ & $\ldots$ \\
\hline $9.993 \pm 0.044$ & $20.350 \pm 0.004$ & $0.134 \pm 0.005$ & $104.4 \pm 1.3$ & 0.137 & 0.038 & & & & & & & & \\
\hline $10.360 \pm 0.047$ & $20.409 \pm 0.005$ & $0.135 \pm 0.006$ & $104.7 \pm 1.4$ & 0.124 & 0.001 & 0.033 & & & & & & & \\
\hline $10.725 \pm 0.068$ & $20.465 \pm 0.005$ & $0.139 \pm 0.008$ & $104.8 \pm 1.9$ & 0.125 & 0.018 & 0.048 & & & $\ldots$ & & & & \\
\hline $11.001 \pm 0.094$ & $20.506 \pm 0.006$ & $0.139 \pm 0.010$ & $106.2 \pm 2.5$ & 0.030 & 0.048 & & & & & & & & \\
\hline $11.269 \pm 0.168$ & $20.537 \pm 0.006$ & $0.139 \pm 0.018$ & $107.1 \pm 4.4$ & 0.025 & 0.037 & 0.119 & & & & & $\cdots$ & $\cdots$ & $\ldots$ \\
\hline
\end{tabular}

Notes.

a Error on the center coordinates defined as Err $=\mathrm{rms}_{\mathrm{fit}} / \sqrt{N}$ with $N \leqslant 128$ the number of fitted points of the isophote.

$\mathrm{b}$ Error of Fourier coefficients defined as Err $=\sqrt{\frac{\sum_{i=10}^{N / 2}\left(a_{i}^{2}+b_{i}^{2}\right)}{N-1}}$.

\section{REFERENCES}

Bell, E. F., \& De Jong, R. S. 2001, ApJ, 550, 212

Binney, J., \& Tremaine, S. 1987, Galactic Dynamics (Princeton, NJ: Princeton Univ. Press)

Binney, J., Gerhard, O., \& Silk, J. 2001, MNRAS, 321, 471

Blumenthal, G. R., Faber, S. M., Flores, R., \& Primack, J. R. 1986, ApJ, 301 27

Cappellari, M., et al. 2006, MNRAS, 366, 1126

Chanamé, J., Kleyna, J., \& van der Marel, R. 2008, ApJ, 682, 841

Conselice, C. J., Blackburne, J. A., \& Papovich, C. 2005, ApJ, 620, 564

Corsini, E. M., Wegner, G., Saglia, R. P., Thomas, J., Bender, R., \& Thomas, D. 2008, ApJS, 175, 462

Cretton, N., de Zeeuw, P. T., van der Marel, R. P., \& Rix, H.-W. 1999, ApJS, 124,383

De Lorenzi, F., Debattista, V. P., Gerhard, O., \& Sambhus, N. 2007, MNRAS, 376, 71

De Lucia, G., \& Blaizot, J. 2007, MNRAS, 375, 2

De Lucia, G., Springel, V., White, S. D. M., Croton, D., \& Kauffmann, G. 2006, MNRAS, 366, 499

Gebhardt, K., et al. 2000, AJ, 119, 1157

Gebhardt, K., et al. 2003, ApJ, 583, 92

Gerhard, O., Jeske, G., Saglia, R. P., \& Bender, R. 1998, MNRAS, 295, 197

Gerhard, O. E., Kronawitter, A., Saglia, R. P., \& Bender, R. 2001, AJ, 121, 1936

Godwin, J. G., Metcalfe, N., \& Peach, J. V. 1983, MNRAS, 202, 113

Gunn, J. E., \& Gott, J. R. I. 1972, ApJ, 176, 1

Jørgensen, I., Franx, M., \& Kjærgard, P. 1995, MNRAS, 273, 1097

Jørgensen, I., Franx, M., \& Kjærgard, P. 1996, MNRAS, 280, 167

Kormendy, J., \& Freeman, K. C. 2004, in IAU Symp. 220, Dark Matter in Galaxies, ed. S. D. Ryder, et al. (San Francisco, CA: ASP), 377

Kronawitter, A., Saglia, R. P., Gerhard, O. E., \& Bender, R. 2000, A\&AS, 144, 53

Mehlert, D., Saglia, R. P., Bender, R., \& Wegner, G. 2000, A\&AS, 141, 449
Mehlert, D., Thomas, D., Saglia, R. P., Bender, R., \& Wegner, G. 2003, A\&A, 407,423

Navarro, J. F., Frenk, C. S., \& White, S. D. M. 1996, ApJ, 462, 563

Persic, M., Salucci, P., \& Stel, F. 1996a, MNRAS, 281, 27

Persic, M., Salucci, P., \& Stel, F. 1996b, MNRAS, 283, 1102

Press, W. H., Teukoslky, S. A., Vetterling, W. T., \& Flannery, B. P. 1992 Numerical Recipes in FORTRAN 77 (2nd ed.; Cambridge: Cambridge Univ. Press)

Saglia, R. P., Burstein, D., Baggley, G., Davies, R. L., Bertschinger, E., Colless, M. M., McMahan, R. K., Jr., \& Wegner, G. 1997, MNRAS, 292, 499

Schwarzschild, M. 1979, ApJ, 232, 236

Simien, F., \& De Vaucouleurs, G. 1986, ApJ, 302, 564

Springel, V., et al. 2005, Nature, 435, 629

Terlevich, A. I., \& Forbes, D. A. 2002, MNRAS, 330, 547

Thomas, D., Maraston, C., Bender, R., \& Mendes de Oliveira, C. 2005a, ApJ, 621,673

Thomas, J. 2006, PhD thesis, Univ. of Munich

Thomas, J., Jesseit, R., Naab, T., Saglia, R. P., Burkert, A., \& Bender, R. 2007a, MNRAS, 381, 1672

Thomas, J., Saglia, R. P., Bender, R., Thomas, D., Gebhardt, K., Magorrian, J., Corsini, E. M., \& Wegner, G. 2005b, MNRAS, 360, 1355

Thomas, J., Saglia, R. P., Bender, R., Thomas, D., Gebhardt, K., Magorrian, J., Corsini, E. M., \& Wegner, G. 2007b, MNRAS, 382, 657

Thomas, J., Saglia, R. P., Bender, R., Thomas, D., Gebhardt, K., Magorrian, J., \& Richstone, D. 2004, MNRAS, 353, 391

Trager, S. C., Faber, S. M., Worthey, G., \& González, J. J. 2000, AJ, 119, 1645

Valluri, M., Merritt, D., \& Emsellem, E. 2004, ApJ, 602, 66

van den Bosch, R. C. E., van de Ven, G., Verolme, E. K., Cappellari, M., \& de Zeeuw, P. T. 2008, MNRAS, 385, 647

Wechsler, R. H., Bullock, J. S., Primack, J. R., Kravtsov, A. V., \& Dekel, A. 2002, ApJ, 568, 52

Wegner, G., Corsini, E. M., Saglia, R. P., Bender, R., Merkl, D., Thomas, D., Thomas, J., \& Mehlert, D. 2002, A\&A, 395, 753 